\title{
Genomic instantiation of consciousness in neurons through a biophoton field theory
}

\author{
Lleuvelyn A. Cacha \\ Department of Psychology \\ Sunway University, 46150 Petaling Jaya \\ Selangor Darul Ehsan, Malaysia \\ lleuvelync@sunway.edu.my \\ Roman R. Poznanski* \\ Laboratory of Biological Modeling \\ The Rockefeller University, New York, NY 10065-6399 USA \\ rpoznanski@rockefeller.edu
}

[Received 10 April 2014; Accepted 14 May 2014; Published 2 July 2014]

A theoretical framework is developed based on the premise that brains evolved into sufficiently complex adaptive systems capable of instantiating genomic consciousness through self-awareness and complex interactions that recognize qualitatively the controlling factors of biological processes. Furthermore, our hypothesis assumes that the collective interactions in neurons yield macroergic effects, which can produce sufficiently strong electric energy fields for electronic excitations to take place on the surface of endogenous structures via alpha-helical integral proteins as electro-solitons. Specifically the process of radiative relaxation of the electro-solitons allows for the transfer of energy via interactions with deoxyribonucleic acid (DNA) molecules to induce conformational changes in DNA molecules producing an ultra weak non-thermal spontaneous emission of coherent biophotons through a quantum effect. The instantiation of coherent biophotons confined in spaces of DNA molecules guides the biophoton field to be instantaneously conducted along the axonal and neuronal arbors and in-between neurons and throughout the cerebral cortex (cortico-thalamic system) and subcortical areas (e.g., midbrain and hindbrain). Thus providing an informational character of the electric coherence of the brain - referred to as quantum coherence. The biophoton field is realized as a conscious field upon the re-absorption of biophotons by exciplex states of DNA molecules. Such quantum phenomenon brings about self-awareness and enables objectivity to have access to subjectivity in the unconscious. As such, subjective experiences can be recalled to consciousness as subjective conscious experiences or qualia through co-operative interactions between exciplex states of DNA molecules and biophotons leading to metabolic activity and energy transfer across proteins as a result of protein-ligand binding during protein-protein communication. The biophoton field as a conscious field is attributable to the resultant effect of specifying qualia from the metabolic energy field that is transported in macromolecular proteins throughout specific networks of neurons that are constantly transforming into more stable associable representations as molecular solitons. The metastability of subjective experiences based on resonant dynamics occurs when bottom-up patterns of neocortical excitatory activity are matched with top-down expectations as adaptive dynamic pressures. These dynamics of on-going activity patterns influenced by the environment and selected as the preferred subjective experience in 
terms of a functional field through functional interactions and biological laws are realized as subjectivity and actualized through functional integration as qualia. It is concluded that interactionism and not information processing is the key in understanding how consciousness bridges the explanatory gap between subjective experiences and their neural correlates in the transcendental brain.

Keywords: Hard problem; consciousness; DNA functioning; subjective experience; biological laws; energy fields; resonance; qualia; solitons; coherent biophotons; interactionism; quantum coherence.

\section{Introduction}

"Science is imagination in the service of verifiable truth.... And in fact, there are some times when imagination leads to a world change".

Gerald M. Edelman

The ultimate understanding of consciousness must reflect upon the emergence of subjective experiences, not at subjective conscious experiences that are being created by the actions and feelings which have no significant bearing on the realizations of raw perceptions of subjective conscious experiences or qualia. The self-referential qualities of consciousness that philosophers call the experiential and subjective aspects occur in conscious life that appears to recognize "self" or self-awareness as opposed to any reflexive organism's behavior is here referred to as consciousness. Subjective experiences are contingent on consciousness yet require complex interactions to take place across scale.

Subjective experiences belong to the realm of quantum phenomena including first-person ontology. They arise from complex interactions at the unconscious level to the more complex conscious experiences. We define aconscious $^{\mathrm{a}}$ in reference to the emergence of this phenomenological entity as being a biological phenomenon that is not repressed and not recalled to consciousness as is commonly associated with unconscious (Freud, 1915). It should be emphasized that aconscious subjective experiences are not the conscious interpretation of the emerged subjective conscious experiences because interpretation involves cognition, comprising cognitive semantics. Aconscious subjective experiences occur in living beings, but in the majority of cases, such experiences can occur without conscious interpretation. The realization of the subjective conscious experiences from the aconscious subjective experiences is referred to as the hard problem of consciousness (Chalmers, 1995).

The recursive look into the subjectivity of consciousness captures account of "selfhood" its own being, felt and perceived from within hence it is first-person ontology on subjective nature of experience that endures through time. In contrast, there is an epistemological view which corresponds to third-person form of knowing. It focuses on physical concepts that have objective descriptions involving biological and physiological knowledge. Further, physical conceptualization also includes

a This name was originated by Franco Orsucci. 
psychological knowledge (such as findings from psychophysical experiments) whose attribution do not contain mental descriptions. Consequently, philosophers construed this knowledge argument that there is an explanatory gap in introspecting first- and third-person ontology (Levine, 1983). It is only a "gap" in the sense that there is only a rudimentary understanding between subjective experiences and the neuroscientific or objective descriptions of those conceptual self-referential experiences. This can be reaffirmed as how a transcendental brain that is non-experiential can fathom subjective experiences that have first-person ontology, such as redness; whereas dynamics and structure refer to third-person ontology, such as detection and discrimination of long wavelength light. To put it differently, the phenomenal concept to explain the experiential aspect of mind (Carruthers \& Veillet, 2007; Levin, 2006, 2008) appears as a quick-fix solution to the mind-body problem defined to be the problem about how phenomenal concepts like subjective experiences and qualia relate to the brain. First-person ontology such as subjectivity, creativity, feelings, intelligence, self-awareness and intentionality does not need an explicit mentioning of the mind, since it includes subjective experience as a fundamental property of the functioning of the brain that is performed through operations that have causal relations to physical processes that have an objective nature or thirdperson ontology.

Consciousness is quite subtly characterized to be reducible to physical processes at either quantum level or at the level of large-assemblies of populations of neurons. At the nanoscale, Hameroff \& Penrose (2014) have hypothesized quantum gravity effects inside microtubules known as orchestrated objective reduction (Orch-OR) theory associated with quantum vibrations in microtubules - the primary correlate of description of consciousness. At the mesoscopic scale, Tononi (2012) hypothesized consciousness as resulting from large-scale integration of information in the brain resulting from its complex neuronal connections of large assemblies of specially selected groups or populations of neurons (Edelman \& Tononi, 1995). However, both reductionist models and information theoretic models of consciousness (Balduzzi \& Tononi, 2009; Tononi, 2008, 2010, 2012; Tononi \& Sporns, 2003) are problematic. The Orch-Or theory cannot explain the spontaneity of consciousness that requires quantum entanglements (i.e., a connectedness of states, such as electron spins) to successfully bridge the gap between nanoscale and large-scale neural network events in the brain; while the information theoretic models explain the qualitative character of experience rather than consciousness itself (Peressini, 2013). Moreover, if consciousness is a biological phenomenon that emerges from the functioning of the brain that supervenes upon the dynamical processes originating in the brain and realized in brain structures (Searle, 2000), then the information theoretic framework of consciousness is flawed because there is a conflict in epistemology.

Searle (2007) refers to consciousness as a biological phenomenon which he labeled as "biological naturalism" to explain in naturalistic terms that there is nothing in the brain that does not supervene upon the physical processes. The basic ideas in biological naturalism are: 
(a) Consciousness cannot function causally in the production of physical behavior (i.e., consciousness has no causal powers);

(b) Neuronal processes can function causally in the production of consciousness (i.e., consciousness is reducible to neuronal processes);

(c) Consciousness is ontologically irreducible (i.e., consciousness has a first person ontology).

Under (a) the firing of a neuron can always be explained in terms of the firing of other neurons, which will be added to all of the other inputs of that neuron, to formulate behavioral responses to those firings, but not as a result of a subjective experience. To put it together, processes such as neuronal firing leads to functions that are subjective processes such as experience of painfulness and these experiences do not interact directly with physical processes such as firing of pain related neurons. Under (b) consciousness is an ordinary physical system that is somewhat conscious or perceived as self-sensation as opposed to a separate property that the brain "gives rise to". For instance, the opening of ligand-gated ionic channels in the postsynaptic membrane is reversibly blocked by anesthetics resulting in loss of consciousness. The difference between property dualism and biological naturalism is that (c) entails that consciousness is a nonphysical emergent property of the brain that is "over and above" the neuronal phenomena, while Searle (2007) advocates that it is a brain state. Biological naturalism has been extended to neurobiological naturalism by Feinberg (2012).

Equally important, is the argument against biological naturalism, the causal powers of large-scale integration in the brain can support dualism because if it would be possible to reproduce artificial consciousness which would imply a dualistic view of the nature of causal powers (Haugeland, 1980). However, Chalmers (1996) claims that subjective experiences are determined by, but not identical to, the functional organization of physical processes in the brain. Therefore if large-scale integration in the brain were to be reproduced artificially as a requirement for intrinsically connecting with the physical elements inherent in the brain, then it would suggest that subjective experiences cannot be expressed as suggested by biological naturalism. In the light of Chalmers' (1996) nonreductive functionalism, subjective experiences supervene upon the physical processes in the brain, like the function of ligand-gated ionic channels supervene upon the physical processes such as ionic transmission across membranes. In nonreductive functionalism, the relevant functions can be realized in one substrate as opposed to multiple substrates. Therefore the limitation of multiple realizability which is an essential tool rather than a thesis of functionalism, opens the possibility of the same function being realized by different structures, but multiple realizability is not an explanatory theory of nonreductive functionalism of mental states meaning that subjectivity supervenes upon the physical processes in the brain. Functionalism is against such multiple realizations. In nonreductive functionalism, subjectivity is irreducible to individual functions arising in the aconscious and therefore it binds into a seamless whole as a functional field that allows for subjective experiences to be recalled to consciousness as qualia. This functional field is not a vitalist concept of 
function as a teleological field that is independent of the structure and intrinsic activity (dynamics) of the system. The functional field is not a "conscious mental field" in the sense of Libet (1996) which would emerge as a function of neural activities in the brain and it would have the attribute of a subjective conscious experience. Rather it is the resultant effect of qualia in the aconscious attributed to the dynamical interactions of brain processes.

An understanding of the role that physical processes have in subjective experiences and the extent to which these processes play in subjective conscious experiences requires an understanding of the following:

(a) Computational models of brain functioning (Izhikevich \& Edelman, 2008; Eliasmith et al., 2012; Stewart \& Eliasmith, 2014) lack subjective experiences and therefore the possibility for sentient awareness (MacGregor, 2006);

(b) Modeling subjectivity through computational neuroscience (Taylor, 2009) is problematic since it defies the existence of the hard problem;

(c) There are conceptual problems of highly conceivable and realistic single neuron consciousness models (Edwards, 2005; Sevush, 2006; LaBerge \& Kasevich, 2007);

(d) The creation of brain models that reconcile all possible knowledge, at present and in the future, en route for continuing improvement in accuracy until eventually the "brain in a supercomputer" model will encounter series of problems with reductionism (Feinberg, 2012).

Information processing is governed through dynamics of physical interactions that transform the information into a new format. This argument of information theoretic integration is that physical signals that carry information, which is not conscious, is accomplished through interactions between physical signals in the third-person ontology, where all information is dynamic and the dynamical interactions lead to a dynamic core (Tononi \& Edelman, 1998), and that is a problem because information processing depends on a specified algorithm a priori.

Subjective experiences are asserted as byproducts of mental processes that emerged from or realized by complex interactions in the brain. It is important to realize that the sources of consciousness are intrinsically intertwined with functioning of the brain and understanding how these self-referential qualities emerge from neural events in the brain is a first step in solving the hard problem of consciousness and ultimately the mind-body problem. It was Chalmers (1993) who first suggested that consciousness requires a foundational theory and that theory is likely to be one that combines both qualitative and quantitative elements (Seth et al., 2006). However, before any such theory can be developed, there needs to be a model in which subjective experiences can be articulated from the emerging physics of consciousness (Tuszynski, 2006). To make progress requires us to solve the hard problem by not reducing qualia to the objective entities of neural processes, but rather by including qualia as part of a theoretical model where subjective experiences are assumed to emerge from neuronal processes.

In a recent review article, Feinberg \& Mallatt (2013) assert that consciousness evolved over half a billion years across phyla of vertebrates and genes someway 
"pattern" the proposed elements of consciousness through neural interactions. Unfortunately their work avoided the intricacies of cognition and quantum-based approaches to consciousness. In this paper, we accept the evolutionary and genetic origins of consciousness and go further by discribing the subcellular loci where consciousness originates and how it may occur in the brain as quantum biological processes. We do not make the category error and say atoms or subatomic particles are conscious, but we proclaim that consciousness emerges from quantum phenomena moving upward to neural networks and systems of networks. Furthermore, we discuss how subjectivity in neocortical functioning is connected with consciousness actualized as self-awareness. Thus our purpose is to give a conceptual underpinning of how subjective experiences arise from neural processes as being dependent upon the complex interactions resulting from quantum events giving rise to a "stream" of consciousness.

\section{A Triple-Aspect Monistic Model in Terms of Dynamics, Selectionism and Cognitive Function}

Subjectivity seems to originate at a different interface between natural understanding and logical formulation -between quantum electrodynamical interactions in deoxyribonucleic acid (DNA) molecules and the residual complex interactions resulting from such events, which serve as the carriers of subjectivity. In order to point to a causal physical link between subjective experience and neocortical function a model of the brain is proposed. The theoretical framework is built upon interactionism through the segregation of subjective concepts from physical properties and their entanglement as functional configurations. A quantum description of the brain as a complex adaptive system was first published by Ricciardi \& Umezawa (1967).

Here we consider a triple-aspect monistic model that has profound association of three aspects that are inseparable (spatiotemporally), yet phenomenologically segregated. This triple aspect monism views that some entities constitute (i) uncognized functions of the self-referential organization consisting of subjective concepts, (ii) cognizable brain functions of the functional organization consisting of functional concepts and (iii) cognitive semantics of the relational organization consisting of physical concepts. The interrelationship between the three aspects is "sui generis". It cannot be causal in the ordinary sense of "efficient causation" (as the action of forces between two bodies) because the aspects are not spatiotemporally separated across any level. Peirara Jr. (2013) referred to these interactions as "sensitive" and "affective". Most philosophers call the interaction from relational to functional organization as "causation" and understanding these interactions is referred to as the mind-body problem, while interactions from self-referential to functional organization are referred to as "realization" and such is also claimed to be the hard problem of consciousness (Chalmers, 2006). The possibility that this model could imply non-causal interactions between the three aspects should also be considered probable. The interconnectedness of the three organizations (hierarchically functional, self-referential and relational) constitutes a triple-aspect monistic model of the brain (see Fig. 1). 

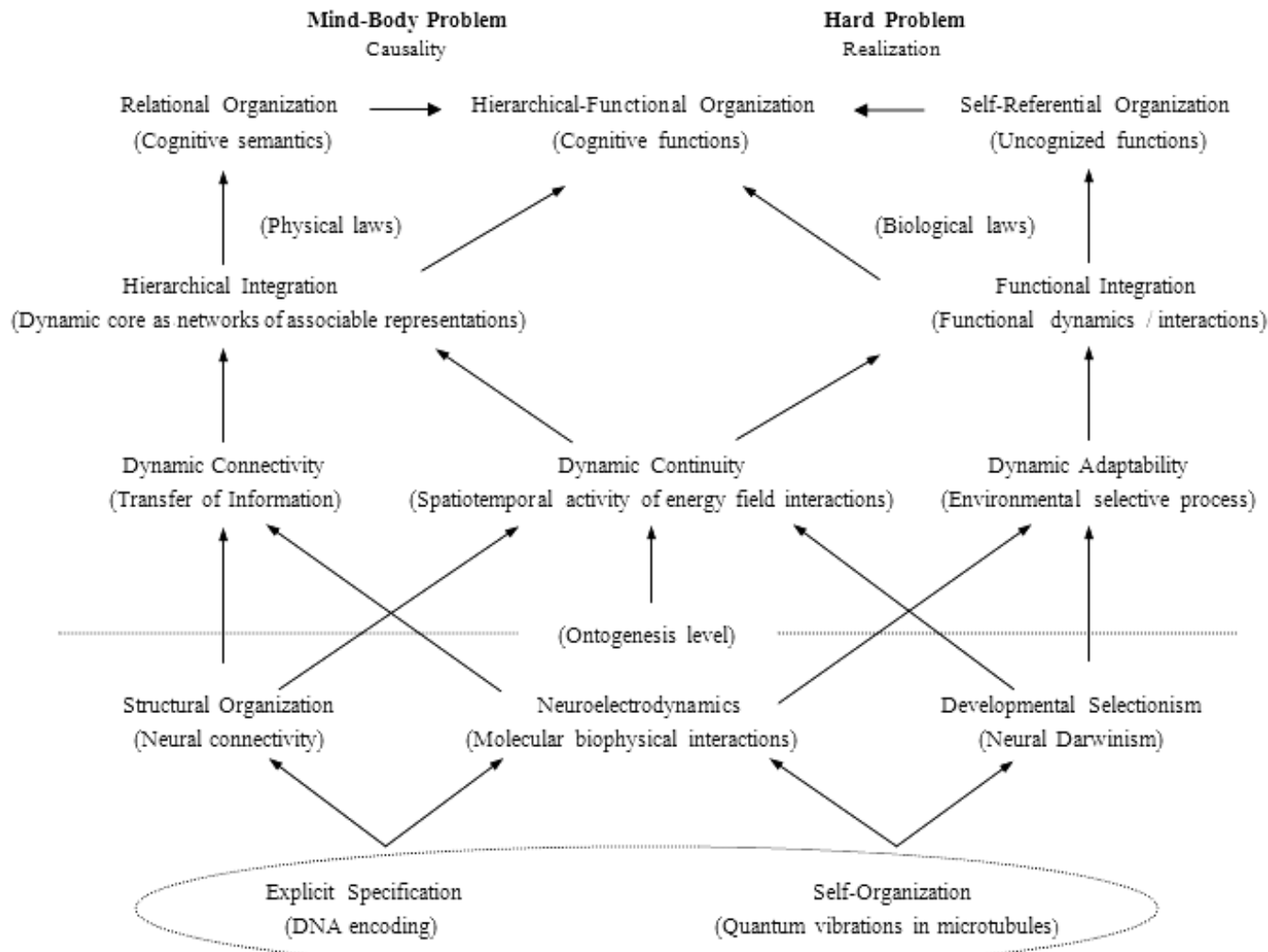

Fig. 1. A triple-aspect monistic model of the brain as a complex adaptive system. Subjective experiences are expressed through a self-referential organization that realizes conscious subjective experiences or qualia through a hierarchically based functional organization which is causally augmented with a relational organization that supports semantics stored in the neural processes of the brain. Relational organization refers to the physical concepts like semantics and functional organization refers to $\operatorname{cog}$ nizable concepts like qualia, while self-referential organization refers to uncognized concepts like subjective experiences. Hierarchical integration (i.e., across scale) occurs during development (i.e., ontogenesis) indirectly affecting structural neural connectivity by modulating dynamic connectivity and by producing a new dynamic process - dynamic continuity enhanced by energy field interactions in neurons and across synapses. Post-ontogenically dynamical connectivity, dynamical continuity and environmental influences through dynamic adaptability lead to hierarchical and functional integration. The left side of the chart is associated with third-person ontology, while the right side reflects firstperson ontology. A physical organization of brain function is non-existent, contrary to common beliefs, that brain functions are confined to certain fixed locations in ordinary Cartesian space. Self-organization is assumed to have originated at the molecular level and is therefore observed at higher hierarchical levels to be a random process, but the transition from molecular to cellular hierarchical levels invokes a non-computational or non-algorithmic process that is not necessarily random and often associated with quantum mechanical indeterminacy. The dotted lines between structural organization, neuroelectrodynamics and developmental selection indicate ontogenesis. Self-replication occurs through explicit specification in the offspring via DNA encoding. Explicit specification remains the only brain operation that is hardwired through phylogenetic evolution. Physical processes, biophysical processes, and biological processes are similar kinds of neuronal processes that are dynamical processes. 


\subsection{Dynamics}

The interaction between protein structures imbedded in a nerve membrane or inside a neuron plays a key functional role determining the topological or spatial distribution of proteins and the connectivity of neural networks. During ontogenesis there are complex interactions brought into existence due to ionic concentration differences between different sites of the cytoplasm that harbor endogenous electromagnetic energy fields involving ionic interactions in macromolecules that can store and transfer information. Physical interactions within neurons at the molecular level bring on the integration process (Aur \& Jog, 2010; Aur et al., 2011). This is how causation in the brain allows for physical concepts like cognitive semantics to emerge. There is a continuous electrically charged flow of information that is carried by the electric field across synapses and within neurons. This dynamic continuity is manifested and results in a dynamic field of influence for augmenting cognitive processes in assemblies of neural networks (see Cacha \& Poznanski, 2011). Without dynamic continuity, the integration processes would be impossible since in principle, to integrate across scale, it is essential for dynamic continuity to be capable of transferring information. This is known as neuroelectrodynamics (Aur \& Jog, 2010).

The determination of the dynamic interactions entails traversing hierarchical levels of structural organization, which over time converges to a dynamic core (Tononi \& Edelman, 1998) as networks of associable representations (Cacha \& Poznanski, 2011). Post-ontogenically dynamical interactions of these energy fields inside neurons and across synapses leads to the transfer of information in neural networks, adaptive influences of the environment and what is referred to as dynamical continuity. The establishment of dynamic continuity is central in controlling a more or less ordered albeit malleable post-ontogenic neural organization leading to a hierarchically based functional organization which supports multi-hierarchical and distinct function that is influenced by this dynamical continuity in the spatiotemporal dynamics of energy field interactions inside neurons and across synapses. Synapses are essential for dynamic continuity to prevail across large networks of assemblies through the act of changing flow patterns in addition to disrupting the flow pattern in distinct associable representation resulting in a dynamic field augmenting cognitive processes in assemblies of networks. The conceptual interpretation of dynamical fields is that they represent guiding "templates" of the dynamical nature of neural assemblies through changes to dynamical continuity. If a dynamic field results from synaptic interactions and electrical interactions inside and between neurons (non-synaptic), then spiking in neurons maybe insufficient for elucidating high-order cognitive functions. Traversing hierarchical levels of structural organization corresponds to structural discontinuity (i.e., a structural border between different scales). Structural, discontinuities result in the non-locality of the global dynamical field. In a space without structural discontinuity, dynamical fields are local. The non-local process allows for the passage from a structure in which phenomena are local to another. Then, the difficulty of the formalized description results 
from this discontinuity in which dynamical fields are different, but necessary for the transport of the interaction.

Dynamic connectivity refers to the integration between micro-, meso- and macrodynamical interactions across spatiotemporal scales. The frequency of firing at synapses can invoke dynamic connectivity formed by changes to the electrochemical signature that is modulated globally by continuous distributions of spatial patterns of dynamic field activity through volume transmission. This is what is loosely referred to as dynamic connectivity (Breakspear, 2004; Jirsa \& McIntosh, 2007). For instance, a physical correlative in the form of neural activity in the cerebral cortex produces an activity in an overlapping dynamic field so that their integration yields new dynamic connectivity. These changes to electrochemical signature over broad areas of cerebral cortex, both are changing the electrochemical signature, which is local and fast in addition to global and slow to guide the dynamics associated with self-organization of textured neural activity that supports cognition (Freeman, 2005).

The mechanism by which changes to synaptic efficiency en route for synaptic connections bring about dynamic connectivity involves a dynamical process that invokes excitatory changes through synaptic plasticity. Post-ontogenic pressures governing dynamical connectivity could in essence reflect the coordinated firing patterns observed in neural assemblies. It is equivalent in some sense to "re-entry" in the theory of neuronal group selection (Edelman, 1978, 1981, 1987) - a term used to represent dynamic connectivity between neuronal groups of strongly connected neuronal assemblies. However, dynamical connectivity alone is insufficient to describe a non-computational process as advanced by neural Darwinism without adaptive behavior based on selectionism. The hypothesis is that selectivity triggered by neural activity (dependent on the dynamics of the signal and the phenotypic adaptability) produces a hierarchically manifested pressure referred to as "dynamic adaptability". It is characterized by a non-random adaptive pressure (Bruzzo \& Vimal, 2007) for the purpose of creating a function across hierarchical levels of functional organization. Dynamic adaptability as a non-computational process is independent of structural constraints (e.g., involving "wireless" volume transmission) and is an ill-defined process influenced by the environment. Brain functions utilize dynamic adaptability governed by a selective process to integrate functional interactions leading to the formation of a functional field. Dynamic adaptability generates adaptive pressures aided by modulatory environments consisting of "silent-signals" not obviously visible as well as field effects which can modulate the excitability of neural assemblies (i.e., non-local interneuronal interactions at the mesoscopic level of functional organization), and other non-classical forms of signaling (e.g., Harris-Warrick \& Marder, 1991; Bullock, 1993, 1997). The selective process by which this occurs is an open research problem, but if quantum coherence is believed to have a role, then such quantum effects need not traverse across the synaptic barrier, but possibly through the extracellular fluid via the volume transmission mode of electrochemical communication (Fuxe \& Agnati, 1991). The possibility of microtubules via a quantum mechanical effect (Hameroff, 1994a,b) could be an example of dynamic adaptability. Such effects 
in arrays of dendritic microtubules have been associated with "quantum consciousness" (Stenger, 1993; Tuszynski, 2006).

\subsection{Selectionism}

Natural selection has provided us a universal frame of meaning into understanding the human brain. Evolution by natural selection in the context of the brain has been referred to as "selectionism" (Sporns \& Tononi, 1994). Selectionism forms an integral feature of neuronal group selection theory and is a dynamic process that is predominant during brain development (Frank \& Wenner, 1993). Selectionism transcends between "developing" and "mature" neural activities (Sporns, 1997). Its operations are in turn governed by the processes of self-organization, appropriately modeled through synchronization and coherency in synergetics (Haken, 1996). The process whereby self-organization installs "neural" connectivity has been discussed by Willshaw \& von Malsburg (1976). However, the hypothesis that self-organization (not combined with explicit specification) directly sets up synaptic connectivity in brain development is doubtful. Genetic specifications of codes for the replication of instructions are transferred between generations by DNA sequences encoded via explicit specification (Watson \& Crick, 1953). Evidence that neurogenesis is mechanistically defined (Caroni, 1998) makes it strongly plausible that explicit specification is governed by instructions generated by DNA sequence encoding (i.e., involving pre-existing patterns of the extracellular chemical forces and the DNA in the cell nucleus). Explicit specification, however, specifies structures via DNA encoding and does not affect directly the dynamic processes leading to brain functions. It is known that (i) DNA is the chemical basis of heredity (McCarty, 1985), (ii) DNA and genes are genuine units of selection (Crick, 1990), (iii) genes that transmit information between generations require the presence of regulatory interactions that are governed by a more complex mechanism for information flow than proposed by the central dogma of molecular biology (Crick, 1970), (iv) conformational changes in DNA molecules are important for understanding DNA functioning (Frank-Kamenetskii \& Lazurkin, 1974) and (v) DNA is capable of emitting coherent photons (Rattemeyer et al., 1981). This view supports a neural Darwinian ontogeny, but the process during post-ontogenesis is more dynamic rather than purely selective meaning that repeated cycles of a particular functional trait do not result in a permanent bias toward such a trait during the life span of the organism, as it would occur under a purely selectionist model.

Based on neural Darwinism, two distinct processes of selection through variation have been postulated (Edelman, 1987): (i) during embryonic development, neural populations form groups with strong synaptic connections and the environment exerts selectional pressure as a result of diversity, variance and imprecision of the synaptic connections. The majority of anatomical connections are not functionally expressed via so-called developmental selection processes. (ii) After ontogenesis in which both structural and functional modules are continually modified, new groups form through the so-called experiential selection process (Edelman, 1983). A 
corollary of this latter selectionism is that selection applied to neuronal populations in which selection processes shape synaptic connectivity and hence dynamic connectivity (Reeke \& Sporns, 1990; Reeke, 1994). What processes govern selectionist theories? If synapses transfer signals indifferently by a similar chemical modulation, then what determines a weak synapse as opposed to a strong synapse? This question was not fully explored in Edelman's work. If selectionist theories do not require a rule for selecting the appropriate "re-entry" path, then such a process must be assumed to depend on some adaptive behavior. Indeed, Calvin (1998) defined a "Darwinian process" as one "...capable of recursively bootstrapping random novelties into something of quality". Calvin's key point is that depletion of variability would result in the discontinuation of selection and thus must remain an essential component of the neuronal adaptive strategy (Sporns \& Tononi, 1994). Although from the neural Darwinian perspective, the output of the system as a whole is of an adaptive value to the organism (Reeke et al., 1990), in the population approach it is difficult to generalize at the individual neuron level (Edelman, 1995).

The tenet of neural Darwinism is that selection through variance has provided insights to the brain's structural and functional organizations and other processes of perception. It is through variance in neuroanatomy and through neural dynamics that essential features related to brain function are established. Dynamically evolving groups of assemblies of distinct multiple neuronal populations are more likely to be associated with dynamic continuity. It would seem plausible that the formation of neuronal groups during ontogenesis leads to a particular functional trait of the group, and particular groups having overlapping functional specializations (i.e., degeneracy) are selected by pre-defined connections after ontogenesis (Edelman \& Gally, 2001). Gierer (1988) has suggested the possibility of a genetic contribution toward "fine tuning" of neuronal connections, although this is a misconception because DNA encoding through explicit specification is associated with formation of structural changes and not the dynamics that ultimately govern brain function. Genetic evolution is itself the result of selectionist processes involving millions of years (i.e., phylogenetics) and not the life-span of an individual organism (i.e., epigenetics) as specified by neural Darwinism. For instance, DNA duplication errors in a gene SRGAP2 have lead to structural changes enabling greater neural connectivity that may have influenced cognition (Dennis et al., 2012). Therefore the self-referential qualities are not hard wired meaning that qualia are a conscious function that is subject to distortion as for example under hallucinatory drugs. Based on selectionism (Edelman, 1987), cognitive semantics that are fluidly engrained within the neural structure as a dynamic field of influence requires no information processing. Semantics/meanings also involve relational thoughts and their processing in related neural networks. They are represented in brain and involve many brain areas. The related physical (electro-chemical) signals from various brain areas interact and integrate at lower levels. In other words, brain structures, functions and subjective experiences related to semantics/meanings are entangled to form subjective conscious experiences or qualia. 


\subsection{Cognitive function}

Brain dynamics allow monitoring brain activities thus providing a mechanistic understanding of brain functions (i.e., cognitive functions and uncognized functions) to be adaptive to the environment and other pressures specifically tuned to the individual organism or its self-referential properties thereby allowing for subjectivity to emerge. The dynamical interactions in the brain remain predominantly dependent on the structural or physical integration with functional integration being influenced by environment, which is the resultant effect of qualia in the aconscious attributed to such dynamical interactions. To understand subjective experiences as cognized brain functions, a hierarchically driven functional organization actualizes the subjective experiences through the metastability of an evolving dynamical information flow aided by selective and adaptive processes from the self-referential organization. Neural correlates of subjective experiences can be understood in ontological, but not in an epistemological sense therefore cognitive brain functions allow for subjective conscious experience to be actualized and a function can have neural correlates such as determined by fMRI. Putative continuity of dynamical processes across spatiotemporal scales is relevant for projecting cognitive function to bring about hierarchical physical integration due to their recurrent interconnections within neural networks in a quasi-syncytial environment, which undergoes a continuous change via chemical modulation and serves as a dynamical field.

The formation of a relational organization relies on associative memory (i.e., new signals invoking old responses) and in particular on the ability to integrate concepts. In other words distantly related information is organized in long-term memory by integrating concepts to related concepts stored in short-term memory. A relational organization is the information that has been stored and retrieved associated with physical concepts involving cognitive semantics derived from the process of hierarchical integration. Cognitive semantics from the viewpoint of relational organization is semantic entailment in the brain of physical interactions caused by forces. The selfreferential qualities that are expressed in terms of functional integration are actualization of subjective conscious experiences or qualia. Qualia belong to the first-person ontology. Subjective experiences are uncognized manifestations of functional interactions leading to the cognizability of perception of subjective experience. These functional interactions reconcile the epistemological qualities with ontological considerations within a unified hierarchical-functional organization that comes about as a causal account of a relational organization that derives from physical integration or hierarchical integration in which dynamical interactions involving information transfer form associable representations. In living organisms, such physical interactions are compounded by a set of functional interactions that can be quantitatively described in terms of cognitive functions. Cognitive function is from the viewpoint of hierarchical-functional organization defined as functional entailment in the brain of both functional interactions and physical interactions. The physical aspect of brain 
operation is referred to as objective third-person ontology. The functional aspect of the same brain operation is the subjective first-person ontology.

Semantics yield distinct content from both first-person ontology (functional-as functional interactions) and third-person ontology (physical-as neural correlates). Both ontological states reinforce each other however data from both are used with a diversity of meanings. Like, we feel the existence of similar subjective state (firstperson ontology) in others by the presence of our own sensation however, the subjective sensation varies greatly in individuals. The functional aspect enables the realization of subjective experience to be cognizable by coalescing with the hierarchical-functional organization. Subjective conscious experiences are cognizable functions arising from functional integration within a self-referential organization and a hierarchically based functional organization that derives from both functional integration and hierarchical integration. We need to be clear why the term integration can be either hierarchical or functional. At the hierarchical integration stage, all physical information is transformed since dynamic interactions are no longer responsible for information transfer of physical information. The integration of each hierarchical level of functional organization may invoke non-computational procedures, especially from the molecular to the cellular level (Penrose, 1995). One suggestion is microtubules harnessing a quantum effect or quantum coherence preserved across the cortical laminae that could generate a pressure or self-organizing force higher up in the hierarchy (Hameroff, 1994a, 1994b). The integration process of creating functions across each hierarchical level of a neural (structural) organization based on "hierarchical functional organization". How is each level of a functional organization established? It was suggested first by Globus (1992) that neuronal function is produced hierarchically at each level by pressures, cytoplasmic, chemical or dynamic. Other evidence points to a hierarchically driven functional organization created not only dynamically, but selectively (Bauer \& Dicke, 1997). Since functional organization may include a selforganizing process that is both selectionist and hierarchically driven, all functional interactions across various hierarchical levels yield emergent complexities of different functions with distinct characteristics, and therefore through integration form a metastable continuum (Fingelkurts et al., 2009).

Cognitive functioning requires both segregation and integration of information (such as in a main complex in thalamocortical neural networks for consciousness (Tononi, 2008, 2012)) from across spatiotemporal scales, resulting in changes to synaptic connectivity over broad areas of cerebral cortex leading to the formation of dynamic fields of neural activity that form neuronal groups or cell assemblies. Analyzing cognition requires a good grasp of how the brain integrates separated tasks into a coherent function. Higher cognitive functions (e.g., language, thinking, planning, reasoning, problem solving, and free will) are influenced by the electrochemical signature of the neocortex through various cortical connections (e.g., associative, commissural and projection fibers) and pathways defined on the basis of the neurotransmitter used (e.g., cholinergic, serotonergic, dopaminergic and noradrenergic pathways). This electrochemical signature also guides dynamical continuity with 
synaptic transmission at assemblies of neural networks in the neocortex without intrinsically altering the network.

\section{Realization of Subjective Experiences Through Complex Interactions Between Energy Fields}

The underlying complexity of events leading from genes to cognition as functions comparable to metabolism would provide us only with a catalytic process, unifying energy with structure in a unified theory of metabolism (Davia, 2006). There needs to be another layer of complexity in terms of energy fields to understand where and how consciousness originates.

Popper et al. (1993) was first to suggest that the electromagnetic energy wave field could represent the aconscious mental functions based on interactionism. Popper proposed that electromagnetic energy wave field residing in the aconscious is subjective experience capable of interacting with subjective conscious experience through a physical force field acted upon by the electromagnetic energy wave field. Interactionism in this sense does not imply an immaterial-material dualism or substance dualism which might violate conservation laws of physics. In their interpretation of Popper's interactionistic hypothesis, Lindahl \& Arhem (1994) proposed a triple-aspect model with two levels of interactions: the first between a certain spatiotemporal pattern of action potentials and an electromagnetic energy wave field representing the aconscious mind; the other between the electromagnetic energy wave field and the conscious mind representing a quale (Fig. 2).

Others like McFadden (2002a,b) proposed that information from neurons is integrated to form a conscious electromagnetic energy wave field. According to McFadden (2013), such an endogenous macroscopic electromagnetic energy wave field as consciousness can influence brain function. This would suggest consciousness has causal actions on the brain. The alternative is that the magnetic field component



Fig. 2. Sir Karl Popper's triple-aspect monistic model adapted by B. Libet as a conscious "mental" field as functional in nature. The boxes on the right refer to the terminology used here. Adapted from Orsucci (2009) with permission of the publisher (World Scientific Publishing Co. Pte. Ltd.). 
has no causal influence on physical behavior. For example, during sleep, the electrical activity is rampant indicating the existence of a magnetic field, yet consciousness is absent. This indicates that consciousness is not an electromagnetic field as claimed by McFadden (2013). Furthermore, at present there is no in vivo experimental evidence to indicate a viable endogenous microscopic electromagnetic energy wave field. Most if not all the experimental data are based on tissue/brain endogenous magnetic fields (Cifra et al., 2011; Buzsaki et al., 2012). Therefore, it would be unlikely that a magnetic field component of the electromagnetic energy wave field could have a direct role in consciousness because the magnetic field can alter cognitive function as for example during transmagnetic stimulation (cf. Snyder et al., 2003), but since consciousness has no causal powers, and if the flow of electrical charges distributed within neurons generates a dynamic electric field with a low magnetic field considered to be negligible (under quasi-electrostatic conditions) then the dynamics of charges are predominantly by electrical interactions in terms of protein polarization (Poznanski \& Cacha, 2011). This is supported by Lindsay et al. (2004) derivation of Maxwell's equation for neuronal modeling where it was shown that large scale phenomena are usually dominated by magnetic fields and the electric field is less important, while small scale phenomena are usually dominated by electric fields and it is the magnetic field which is less important. This would suggest that an electric energy field has a role in consciousness at the single neuron level.

Our hypothesis assumes that the collective interactions in neurons have sufficient macroergic effect to produce sufficiently strong electric energy fields ${ }^{\mathrm{b}}$ for electronic excitations to take place on the surface of endogenous structures via alpha-helical integral proteins as electro-solitons (Brizhik, 2008). These electro-solitons carry an electric charge moving in-between alpha-helical integral proteins of macromolecules. Similar endogenous microscopic electromagnetic energy wave fields are considered to be propagating in the cavity of microtubules, transporting and carrying information (Sataric et al., 1992). Solitons are self-localized robust and long-lasting solitary waves that do not disperse and preserve their identity as they travel through a medium are ubiquitous in nature. Subsequent radiative relaxation or decay of electro-soliton waves propagating through a medium such as the intracellular space, transfers energy via interactions with DNA in the nucleus to induce conformational changes producing the spontaneous emission of ultra weak, non-thermal radiation referred to as coherent biophotons ${ }^{c}$ (Popp et al., 1984, 1994; Popp \& Chang, 1998). Interaction between the electric field and DNA results in conformational changes to DNA molecules resulting in confined spaces similar to photonic crystal structures that are an ideal substrate for a quantum effects to instantiate coherent biophotons in the vast majority of neurons in the brain which can form a biophoton field since it is

\footnotetext{
${ }^{\mathrm{b}}$ Cortical action potentials of $\sim 1 \mathrm{~ms}$ refractory periods could bring on strong electric fields. This differs with plant cells and cardiac muscle cells having action potentials in the refractory range of $\sim 10 \mathrm{~s}$ and $\sim 250 \mathrm{~ms}$, respectively; while skeletal muscle cells have compatible refractory periods to neurons but they lack gap-junctions or electrical synapses required for a unified field to emerge.

${ }^{\mathrm{c}}$ This name was originated by Fritz-Albert Popp.
} 


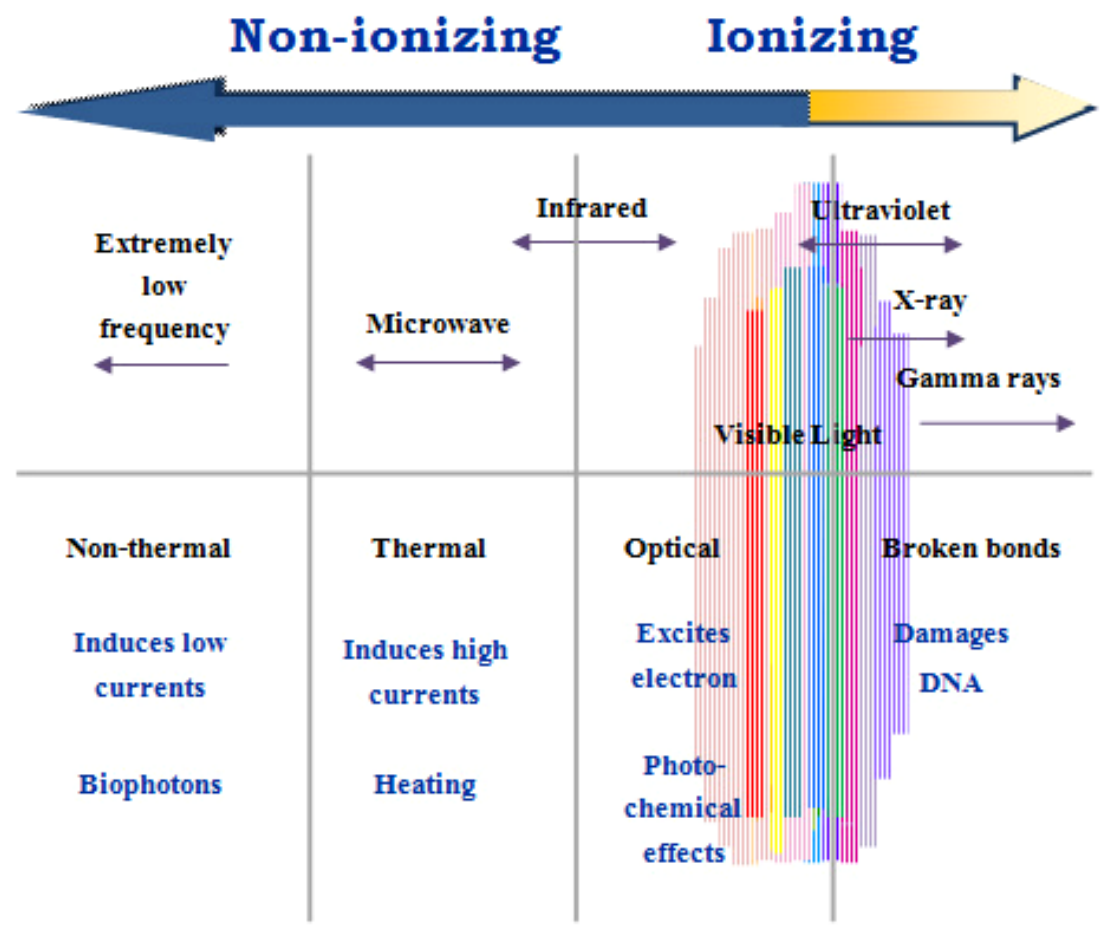

Fig. 3. The electromagnetic spectrum showing the positioning of biophoton emission in the ultra weak, non-thermal and non-ionizing range of the spectrum. The spectral region in which biophotons are emitted ranges in frequencies from $200 \mathrm{~nm}$ to $800 \mathrm{~nm}$ (Popp et al., 1994).

radiation of light there is near instantaneous communication throughout — referred to as quantum coherence. The binding of the biophoton field may involve a Fröhlichtype condensate proposed for any system far from equilibrium surrounded by a thermal bath, with the presence of a non-thermal energy source (Fröhlich, 1968). However, coherent biophotons emissions occur in the non-thermal part of the electromagnetic spectrum at ultra low frequencies (Fig. 3) and is referred to as DNA excimer radiation $(\mathrm{Li}, 1992)$.

The coherence of biophotons stems from the result of electric energy fields propagating in the intracellular space or cytoplasm as solitonic transmission with collective dynamics (Giudice et al., 1988). The electric field does not need to be coherent; it is the intracellular fluid that creates coherency in the presence of an electric field. If biophotons are produced from a coherent field then their frequencies will be more closely correlated than biophotons produced from a non-coherent field from exciplex states of DNA molecular interactions. Coherent biophotons are produced by an electric field and not a random chemical process (Popp et al., 2002). Yet, any such coherent biophoton field generated in neurons would only maintain its coherence for an extremely short time because of decoherence (Tegmark, 2000). What it means is that the emission of coherent biophotons upon interaction with DNA must then re-emit almost continuously before decoherence takes effect in order to maintain the 
biophoton field unless there is a Fröhlich-type condensate or nature has developed photonic crystal structures made up of DNA molecules enabling the instantiation of coherent biophotons in the vast majority of neurons in the brain which can form a biophoton field. If each cell is emitting biophoton field, then the whole living organism is, in effect, a resonating biophoton field - a ubiquitous non-local field. And since biophotons are the entities through which the entire brain can readily communicate information, there is near-instantaneous intercommunication throughout. A biophoton field occurring spontaneously throughout the whole brain will provide an information character of the electric coherence, and hypothetically allow for the possibility of a unified consciousness to emerge (Van Wilk et al., 2007). There are real life examples, which indirectly support consciousness as unified and nonlocalized. For example, children born without cerebral hemispheres remain self-aware of their immediate environment (Shewmon et al., 1999; Merker, 2007). Thus consciousness through self-awareness appears to be possible with only a midbrain and a hindbrain intact. Another example occurs when an anesthetic molecule enters into the lipid phase of the membrane and makes the membrane more fluid-like, which can result in a weak macroergic effect possibly due to lack of action potentials firing that inhibit electronic excitation for the emission of coherent biophotons.

Although coherent biophotons are emitted through quantum mechanical effects upon interacting with DNA, exciplex states of DNA in the cell nucleus of neurons can trigger chemical reactions. The emission of non-coherent biophotons originates from oxidative metabolism (chemical reactions) of exciplex states of DNA as a byproduct of these chemical reactions. These non-coherent biophotons occur in every cell in the body where chemical reactions take place and may play a role in actualizing self-awareness in the brain. Neurons continuously produce non-coherent biophotons during their metabolism (Isojima et al., 1995; Kataoka et al., 2001; Kobayashi et al. 1999; Rahnama et al., 2011) and the source of non-coherent biophotons derives from oxidative metabolism of mitochondria and lipid peroxidation (Thar \& Kuhl, 2004). Excited states of macromolecular structures in neurons can also be a catalytic process generally associated with the presence of an oxidative metabolism that accompanies the production of reactive oxygen species (ROS) which participate in the regulation of a wide spectrum of biochemical and physiological functions. Biophotons are produced by normal chemical processes, just as the well-known phenomenon of bioluminescence. A distinct noncoherent emission of photons as byproducts of metabolism, like thermal radiation and bioluminescence/chemiluminescence caused by radical reactions, oxidation etc such biophoton emission refers to the phenomenon of constant and spontaneous emission due to metabolic activities, without excitation or enhancement. This biophoton emission can also reflect a pathophysiological state with respect to mitochondrial energy (ATP) production and the susceptibility to oxidative stress which is derived from the excessive production of ROS or a lack of activity for antioxidant protection.

How quantum electrodynamics produces this phenomenon which we call selfawareness in the brain? We believe that brains have evolved to utilize both the wave and particle properties of electromagnetic energy fields not only for self-awareness, but 
also to transform objectivity to subjectivity. Here objectivity in terms of electromagnetic energy wave field is regarded as a smooth, continuous field, propagated in a wavelike manner, but transformed into subjectivity through complex quantum interactions of subcellular energy fields, where energy is understood to have a fundamentally subjective aspect in nature. The transition enabling objectivity to be transformed into subjectivity entails representation of DNA codon sequences as frequency patterns of energy fields reflected upon the uniqueness of their braiding patterns that would serve as a "quantum tape" to emulate consciousness; at which upon scanning of the "quantum tape" reveals the self-referential character of the brain through fluctuation of ambient potentials in the electrolytic fluid representing the intracellular domain of neurons (cf., Green \& Triffet, 1997).

The instantiation of coherent biophotons facilitates the conduction of a biophoton field which is in turn realized as a conscious field when it becomes actualized by selfawareness as a result of re-absorption of biophotons by exciplex states of DNA that serve as a "feedback" mechanism to release non-coherent biophotons emissions leading to metabolic activity and energy transfer across proteins in macromolecules as a result of protein-ligand binding (Turton et al., 2014). It entails DNA chromosomes acquiring unlimited information from metabolic activity that in turn produce regulative electric field signals as feedback. This is assumed to be the subcellular loci for the opening for self-awareness that allows for objectivity to have access to subjectivity in the aconscious. In other words, we claim that self-awareness comes to fruition as DNA re-absorbing biophotons in the aconscious where it is recalled to consciousness in a way similar to the role of potentialities describing the passage of potentiality to an actualization. The resultant conscious field that instantaneously is unified through protein conformation changes guiding biophoton communication in neurons (Sun et al., 2010) and in-between neurons through electrical synapses and across synapses in networks of neurons in the cerebral cortex (cortico-thalamic system) and subcortical areas (e.g., midbrain and hindbrain) thus providing an informational character of the electric coherence. The brain is privileged to provide selfawareness not possible in other biological organs where biophotons may be emitted since the conscious field embroils a large number of specifically designated functional areas in the brain that require energy to be actualized in tandem with the traditional electrochemical communication.

The spark of consciousness is a quantum event being the result of quantum coherence through biophoton emission due to DNA re-absorption and energy transfer created by metabolic energy that is stored as a form of coherent excitation. It is these coherent excitations that are considered responsible for generating and maintaining long-range order via the conduction of the energy field interactions which give access to subjectivity occur at the timescale too fast to those based on integration of neural information. For example, neuron firing of action potentials in the millisecond range does not reflect upon the subjective conscious experiences that occur on much faster timescales driven by solitonic transmission on timescale of $5 \times 10^{-7} \mathrm{~s}$ (Sataric et al., 1993 ) as opposed to neural dynamical timescales of $5 \times 10^{-3} \mathrm{~s}$. Yet when protein 
polarization is taken into account, neurons no longer operate too slowly for quantum coherence, dismissing the idea of too short decoherence time of biophotons $\sim 10^{-13}$ sec (Tegmark, 2000).

In an earlier attempt to explain hard problem in terms of quantum consciousness, it was pointed out that quantum effects are insufficient (Smith, 2006, 2009). Energy is intrinsically qualitative so, for example, the qualitative feeling of seeing "red" is a form of physical energy. The uniqueness of frequency patterns of energy fields reflected upon the DNA codon sequences braiding patterns and the regulation of electric field signals from metabolic activity by feedback from DNA chromosomes acquiring unlimited information could therefore suggest that the re-absorption of biophotons by DNA plays a role in what earlier quantum consciousness researchers had not observed. Indeed the resultant conscious field is attributable to the effect of specifying qualia by such a "feedback" mechanism throughout the brain and in specific networks of neurons that are constantly transforming into more stable associable representations as "molecular" solitons. Biophotons are absorbed by DNA

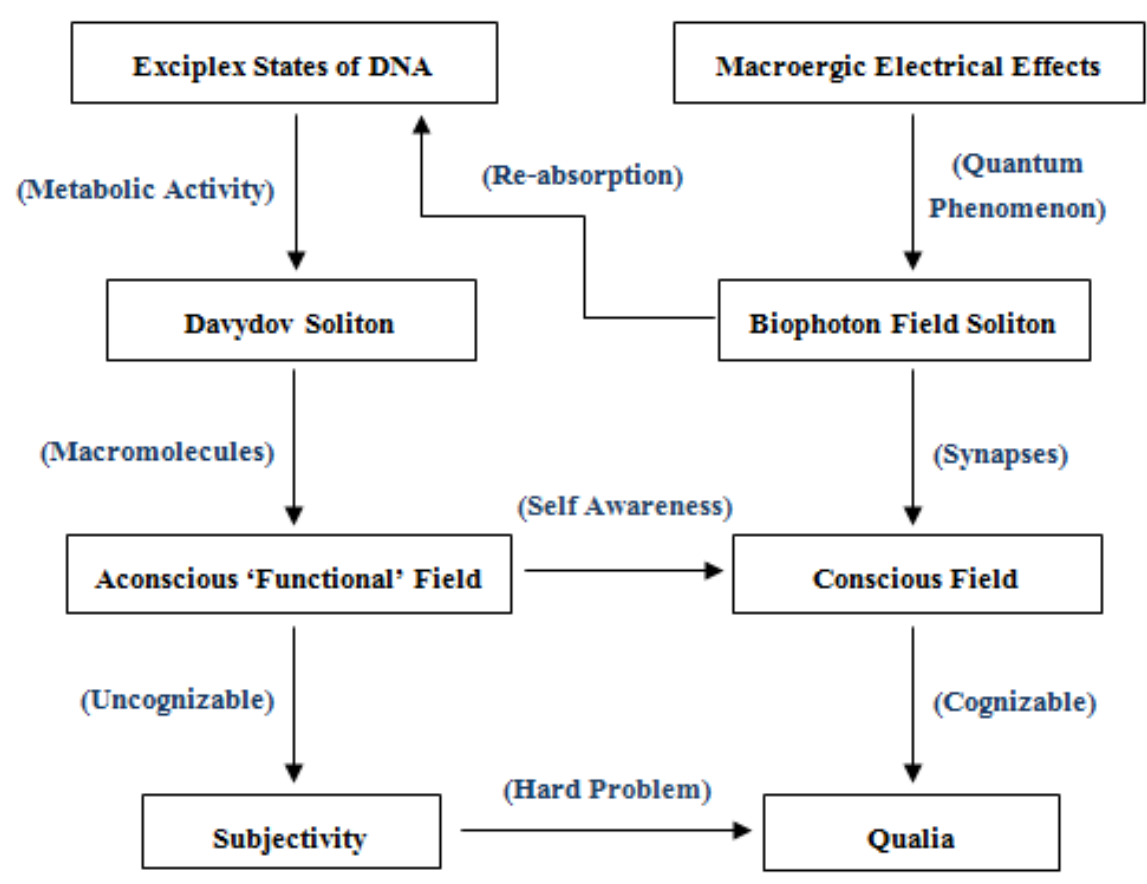

Fig. 4. A schematic flowchart of the neurobiological quantum phenomenon leading to self-awareness through biophoton field as a soliton forming a unified conscious field and metabolic energy activity leading to subjectivity as Davydov solitons in macromolecules forming a functional field in the aconscious. The hard problem is how any objective explanation can explain subjective phenomena given that objectivity seems not to have direct access to such phenomena. Such an indirect access that links the ontologically subjective experience with epistemologically objective concepts is the aconscious. Subjectivity seems to originate at a different interface - between quantum electrodynamical interactions in DNA and the residual complex interactions resulting from such events, and how these modifications to DNA molecules provide the means for photonic crystal-like structures to instantiate coherent biophotons through a quantum effect and subsequently as the carriers of subjectivity. 
producing exciplex states in DNA molecules where intrapeptide vibration amide-1 are excited at an end of alpha-helical protein molecules that release amide- 1 vibrational energy for transport of metabolic activity in macromolecules as Davydov solitonic transmission (Scott, 1985). Davydov solitons (Davydov, 1991) provide self-regulation of metabolic and coherence of the guiding electro-solitons (Brizhik et al., 1998). As well, Daydov solitons are referred to as a "molecular" solitons because they are considered to be moving in between macromolecules that provide the energy and charge transport during metabolic activity (Tuszynski et al., 1984). It should be clear that any external stimulation by light penetration of the cranium (cf. Goodman, 1983) would normally elicit non-coherent biophoton emissions that do not regulate neither the conscious field nor the aconcious functional field as biophotons do not interact whereby any external influences of light that would normally pass through without any effect. The Davydov soliton may play a role in formation of a functional field in the physical observation of subjective experiences in the aconscious from the feedback or reabsorption of biophotons by exciplex states in DNA for eventual recalling to consciousness as qualia. The change from objectivity to subjectivity is a quantum mechanical phenomenon that is brought about by the interaction of an electro-soliton with DNA to spontaneously emit a biophoton field as a soliton, propagating by way of protein-protein biophoton interactions inside neurons and in-between neurons through electrical synapses and across neurons through chemical synapses forming a unified conscious field (Fig. 4).

\section{Metastability of Subjective Experiences Through Matching of Resonant Dynamics}

A unified consciousness either exists at the entire cortical level (John, 2001) or primarily in the thalamocortical region (Llinas et al., 1998). According to McGinn (1995), the difficulty comes from consciousness being nowhere and everywhere in the brain, thus not amenable to the methodic reductionist analysis. Sigmund Freud in 1895 wrote about neural networks driven by non-physiological energy forces that altered conscious processes. Such a Freudian viewpoint can be modernized (Solms \& Turnbull, 2002). For instance, associable representations in the brain, as selectionism maneuvering dynamic continuity by an adaptive pressure from which higher cognitive functions result from such activity in assemblies of neural networks and from which subjective experiences as metastable and uncognized phenomenon are realized as a cognizable phenomenon.

Subjective experiences are the self-referential qualities that we directly experience, and this is regardless of whether we are conscious or unconscious during sleep. This suggests that subjective experiences can be either cognized functions or uncognized functions depending whether one is associated with qualia through consciousness. For example the subjective conscious experience of pain, is a cognizable phenomenon. However, the process of transduction, transmission, and modulation associated with perception of pain, are based on empirical methods that reveal objective physical 
aspects such as neural correlates of various conscious experiences, that do not directly reveal epistemological, self-referential qualities, such as qualia.

Subjective experiences are represented in terms of a functional field that supervenes upon the integration of dynamic interactions and which is a continuum of functions that are hidden from the external world in the aconscious to be recalled to consciousness as qualia through functional integration. Dennett (1991) states that higher-order functions must necessarily supervene upon the lower-order functions. Such supervenience requires the existence of hierarchical levels driven by less and less sophisticated functional organization. No such problem exists if in a particular functional organization there is a continuum of functions as an aconscious functional field. For instance, perception, memory, emotion, thinking and creativity are all functions arising from dynamical interactions in the aconscious. In the same way unconscious handling of information in the brain is uncognized, but becomes cognizable when recalled to consciousness and thus, cognition occurs. Subjective experiences are brain functions that are cognizable, yet no amount of physical information can capture the subjective raw qualities of conscious experience (the qualia) whose meanings cannot be fully understood. Hence there exists an epistemological gulf between descriptions of physical events in the brain and the subjective experiences that are presumed to be associated with those events. Thus, functionalism is not robust enough to explain individual differences in qualia (Block, 1994). A problem with the non-uniqueness of qualia to a particular function is that functionalism asserts subjective experience can be realized independently of the functional field in the brain. This in our view contradicts, the fundamental idea of supervenience that requires no change in the subjective experience without change in the underlying structure. If there is a mapping from a set of self-referential qualities to neurobiological processes, then the functional field realization is uniquely possible.

A functional field can be actualized by brain processes that can have the ability to express subject experience with first-person ontology. Therefore subjective experiences come from functional properties of the brain. And functions arise out of the inter-connectedness of the brain through dynamical interactions. A functional field supervenes upon the dynamic interactions that occur within the neuronal processes of the brain (Freeman, 2007). The mestable dynamic interactions invoking associable representations in brain processes are uncognizable in space and time which means not repressed and not recalled to consciousness, i.e., aconscious which is outside of consciousness and physical processes. The dynamical interactions metastabilize (cf. Kelso, 1995; Fingelkurts \& Fingelkurts, 2004; Freeman \& Holmes, 2005; Fingelkurts et al., 2009) and cognize in the process of realization of the subjective experiences in the brain.

A viable alternative is that functional field supervenes upon dynamical interactions that give rise to Davydov solitons that mediate energy dissipation as a process of catalysis (Davia, 2006). Solitons are a continuous phenomenon, and are localized within a region and can interact with other solitons, and emerge from the collision 
unchanged. Thus the qualitative nature of consciousness relates to correlations of subjective conscious experience supervening upon dynamic and relational properties of solitonic transmission that is realized by localized ontological phenomenon that unifies the epistemological aspects through nonrepresentational cognitive semantics. Such interactions give access to subjectivity spontaneously that do not require an integration process. The objectivity of these dynamical interactions is transformed into subjectivity by a "switch" caused by the wave-particle duality description of quantum mechanics when an electric energy wave field interacting with DNA results in coherent biophoton emissions that are re-absorbed by DNA to produce metabolic activity that can through dynamic ionic interactions involving macromolecules be transposed as Davydov solitons to bring about an aconscious functional field.

The realization of uncognized brain functions into cognized higher brain functions follows the process of resonance (Golant, 1989; Grossberg, 1999, 2007). Resonance is an organizational reservoir of potential changes to neural dynamics without any specific structural re-organization. The concept of resonance in neural systems concerns the topology and includes the tendency for maximum potential of organization. Resonance occurs when bottom-up patterns of neocortical excitatory activity as Davydov solitonic transmission are "matched" with top-down expectations as dynamic pressure that is influenced by the environment. This bottom-up/top-down resonance lasts longer and is more amplified than any individual activation with resonance. The process of matching involves selecting bottom-up signals then what keeps the selected signals from reactivating their top-down expectations in a continuous cycle of bottom-up and top-down interaction. It is suggested that the type of interaction involves higher-order thoughts ("thoughts about thoughts") (Rolls, 1997) or self-recognition (Orpwood, 2007), which for example, can explain painfulness because it assumes that the state of each entity has physical aspect and segregable functional aspect of a state of that entity. When a pain related stimulus activates the pain receptors, feedforward signals are generated. These signals interact with cognitive feedback signals that also have physical and segregable functional aspects. The segregability is the supervenience of the functional on the physical. This functional aspect related to the feedback signal also contains the state related to subjective experience painfulness in superposition with other states related to other subjective experiences. The conscious aspect such as a quale is actualized by an aconscious functional field that supervenes upon the dynamical interactions governed by Davydov solitons. For example, happiness-sadness functions cannot supervene from structure alone; they depend on dynamic interactions, but the feeling of being happy or sad cannot be explained by subjective experiences and must be related to other relational organization that are based on physical laws for recalling to consciousness as subjective conscious experience or qualia. A schematic illustration of the process whereby Davydov solitonic transmission as an associable representation arising from the aconscious form qualia is shown in Fig. 5 . 


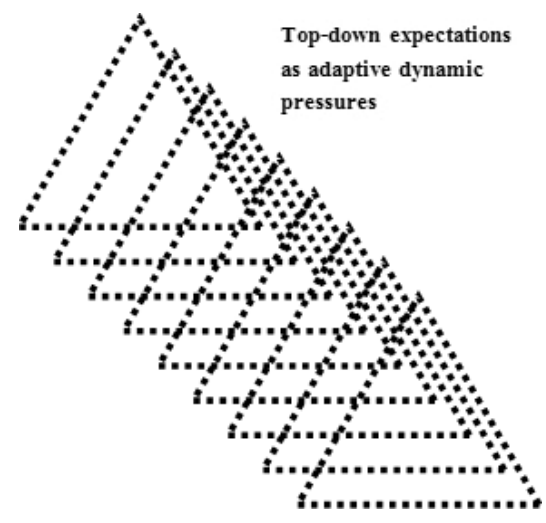

(a)

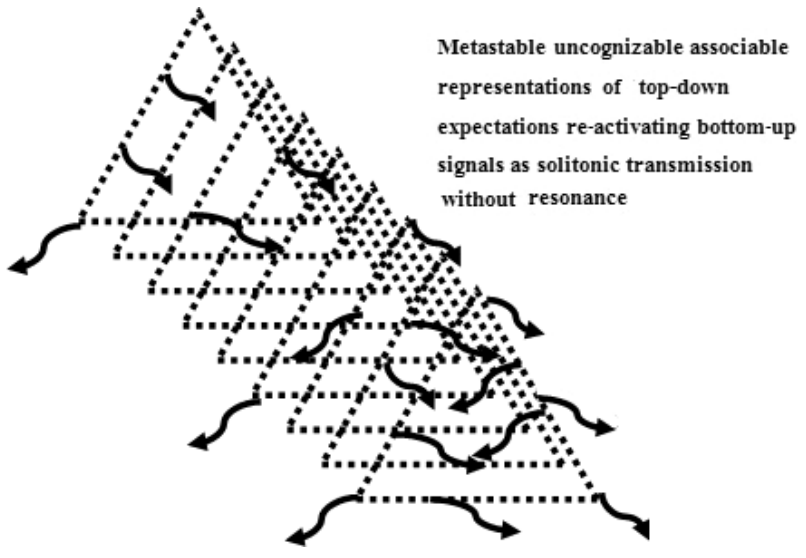

(b)

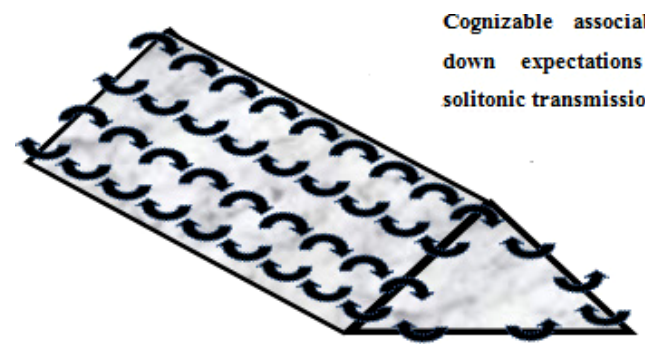

(c)

Fig. 5. A schematic illustration of solitonic transmission as a dynamical associable representation of subjective experiences arising from the aconscious to form qualia. Subjective experiences as a resonant phenomenon that emerge as a functional field whereupon "matching" of top-down (feedback signals) expectations and bottom-up (feedforward signals) processes during the attainment of a self-referential organization through dynamic adaptability. (a) The learned expectation of a triangular representation from top-down adaptive pressures as a discontinuous set of discrete events in time that is incognizable. (b) Without resonance, there is inadequate matching of the top-down adaptive pressures and the bottom-up neural signals. The solitonic transmission results in a highly metastable and uncognizable associable representation of a subjective experience as indicated by the arrows. (c) Feedback relationships among neurons by way of adaptive resonance unifying top-down expectations with bottom-up solitonic transmission of neural signals that result in an associable representation of a quale. The dissipative solitonic triangle is not a representation, but an ontological phenomenon that unifies the epistemological aspects as resonance develops. The characteristics of the dissipative soliton are of molecular origin involving metabolic energy (Davydov, 1973, 1991). Adapted from Davia (2006) with permission from the author.

\section{Selection of Subjective Experiences Through Functional Interactions and Biological Laws}

The functional field supervenes on the dynamical interactions involved with dynamical continuity and dynamical adaptability as a Davydov soliton, which leads to the "emergence" of a unified experience of "red object slowly moving from left to right" through resonance. It is emphasized that subjective experience is the realizer of the functional field, not the functional field itself which means there is a process 
leading to the functional field. This integration of functional interactions entails selection of specific subjective experiences. There are functional interactions in each area for the detection and discrimination of function related to each of motion and color. Then there are functional interactions between two related functional units. The integration of all these functional interactions forms an aconscious functional field of an unified experience of 'red' moving object.

The brain as a biological organ has a physical aspect because of its structure and a biological aspect because of its function (Chauvet, 2004). The physical aspect concerns with physical interactions relating the structural organization of matter without affecting its functional organization. Biological aspects are an abstract description of the underlying functional processes in terms of a set of functional interactions. In supervenience, biological aspect cannot be changed without altering some physical aspect. The related concept of physical realization involved no biological properties that can have nonphysical realizations (Kim, 1998), being compatible with functionalism. We use concepts such as "emergence" (Chalmers, 2006), "supervenience" (Kim, 2009) to explain subjective experiences that may appear to be less mysterious as long as both concepts are appropriately "unpacked" in terms of selection. Let us take an example of "red object slowly moving from left to right". There are three physical attributes, namely velocity and direction of motion and long wavelength reflected light representing red color. There are two functions, namely, detection/discrimination of motion and that of color. Signals from retina travel to V1 and eventually get segregated to neurons of color area V4/V8/VO and motion area V5/MT for specific analysis (Vimal, 2008, 2010).

Subjective conscious experience can only be conceived under specific conditions regardless of the extent of knowledge garnished on the complexity of the operational mechanisms. First, the existence of functional interactions is the first condition for conscious self-experience in nonliving matter. Whereas the action of physical forces, which are symmetric in nature, lead to structural stability in nonliving matter, the existence of functional interactions leads to an increase in specialization and hierarchization resulting in a decrease in resonance. Finally the integration of functional interactions must allow for the propagation of non-local functional fields according to the relationship between the topology and the geometry of the neural system.

Even though this does not imply that all living organisms support qualia, it is obvious that rudimentary forms of self-awareness exist in lower forms of life (Feinberg \& Mallatt, 2013). Clearly from the perspective of evolution of living matter there is an additional characteristic which is required for qualia. The consensus is for biological organisms to behave like complex adaptive systems with ability for selfreplication of functional interactions. To understand complex adaptive systems, one needs to understand how models can induce the topology of the biological system. Using topological methods, Rashevsky (1961) was able to analyze the functional organization of any living system to understand biological laws. One such biological law is the idea of self-replication of functional interactions that brings about their integration in terms of a functional field. This distinguishes a conscious organism 
from an artificial system. In an artificial system, like a robotic machine with a supercomputer brain, every function is entailed by another function, thus an infinite regress exists, but in conscious organisms the infinite regress does not exist when there is self-replication of functional interactions (Rosen, 1991). It is therefore charlatanism to conceive conscious robots.

Brain structure is made of organic matter and encapsulated within skulls of higher vertebrates that evolved consciousness through 500 million years of natural selection (Feinberg \& Mallatt, 2013). Brains are part of the evolutionary nexus that is governed by both non-heredity and DNA self-replication through explicit specification mechanisms that allow the self-referential character of the brain to express subjective experiences. Therefore subjective experiences are more than self-awareness in some vertebrates or pure reactions in invertebrates. Evolution theory facilitated by degeneracy in biological systems (Edelman \& Gally, 2001) represents a conceptual idea in understanding how subjective experiences in neuronal systems evolved by natural selection. Thus our premise is the necessity of biological laws as an evolutionary stratum applied to living matter over physical laws for nonliving matter.

Biological laws apply to all living organisms made of cells having evolved from physical processes and are not only restricted to excitable cells of the nervous systems. Accordingly living organisms obey biological laws of organization and functioning that differ from physical laws in nonliving matter. The structural organization of nonliving matter is deduced from the combination of forces acting on elementary physical structures. In living organisms, such physical interactions are compounded by a set of functional interactions that enhance the reliance on information transmission without the processing of information. Living organisms are open systems with continuous exchange of energy with the environment and are intrinsically heterogeneous; they possess a property of self-organization and selfreplication. They are in a state far from thermodynamic equilibrium. Their evolution is phylogenetic and not epigenetic because upon the achievement of complete thermodynamic equilibrium, all the proteins of a living organism disintegrate. In nonliving matter, thermodynamic entropy cannot but increase with time, leading to an increase in molecular disorder. As such it follows that biological laws cannot be reduced to physical laws, but since biological laws are a compounded form of physical laws, they cannot "differ" from physical laws because physical laws cannot be violated.

Schrödinger (1944) was the first person to suggest that new laws are expected in biological or living organisms:

"... in short, that from all we have learnt about the structure of living matter, we must be prepared to find it working in a manner that cannot be reduced to the ordinary laws of physics".

Biological laws describe the right compromise between complete order and complete randomness. What this means is that some unpredictability allows biological 
laws to kick in. If there is too much order from complex biochemical interactions to the evolution of organisms and even through to the functioning of the brain, then the emergence of consciousness could not take place. Likewise, if there is too much randomness from complex biochemical interactions to the evolution of organisms and even through to the functioning of the brain, then the emergence of consciousness could not take place. This suggests that the resolution of subjective experiences in neocortical functioning cannot be emulated without an understanding of the unpredictability of biological laws. In nonliving matter, the biological aspect which constitutes function that potentially leads to the capability of expressing subjectivity is latent since nonliving matter is inert. (This excludes some forms of energy in Nature.) Living organisms such as mammals and in particular primates have hierarchically driven functional organization that is sufficiently complex to sustain subjective experiences. Biological laws govern the complexity for the realization of subjective conscious experiences. In the absence of highly complex synaptic connections among neurons with rich dynamics, it would be unlikely that subjective experience exist since the functional interactions would be seriously underdeveloped, most likely latent and limited to quantum-generalized processes.

Fundamental characteristics of living organisms such as asymmetry and inhomogeneity are well-known examples of laws' (Chauvet, 1993c,d). Biological laws explicitly include the property of nonsymmetry (Chauvet, 1993c) between the sources and sinks involved in functional interactions and the property of non-locality in its dynamic organization (Chauvet, 1993d). The nonsymmetry of the action from source to sink, which implies a local transformation in the sink, and the non-locality of the action in space, which arises from the hierarchical structure. Biological nonlocality is the representation of activity and how it results from the continuity in both space and time, underlying hierarchical physical integration. So, the hierarchy describes a specific physiological process at each level, coupled with processes evolving in adjacent levels. Chauvet (1993a,b) has proposed to describe these physiological systems in terms of the nonsymmetric and non-local interactions from sources to sinks, with transformations in the sink which correspond to distinct dynamical fields. This is the case of action potentials from neuron to neuron at synapses and is referred to as hierarchical integration (Chauvet, 1993a). Rosen's relational biology (Rosen, 1958, 1991, 1998; Rashevsky, 1961) has been extended by Chauvet (1996) to include concepts of hierarchical and functional integration.

For instance, if the product of a functional interaction such as an ion, destined to modify, for example, the ionic charge of a molecule, moves from a source to a sink, then there must exist some mechanism of transport, e.g., an ionic gradient. Thus, the concept of functional interaction involves, in mathematical terms, the action of a field operator on a variable emitted by the source. The field operator acts on the variable, the value of which undergoes a transformation because of the source. Functional interactions have a role analogous to that of a force in physical interactions with symmetry and locality in place. Forces can only operate when the medium is homogeneous (Chauvet, 2002). Living organisms contain structural discontinuities 
between which functional interactions take place. Functional interactions induce the topology of the biological organism, i.e., the functional organization of the biological organism (Chauvet, 1996, 2002). Topology considers space and time and continuity, while geometry influences the dynamics of the process, corresponding to the spatiotemporal functional organization. Thus geometry as a metaphor for qualia (Balduzzi \& Tononi, 2009) does not entail the presence of dynamic continuity and hence there can be no integration of information. It may be that some physical systems have dynamic continuity, but they lack the property of nonsymmetry, which is referred to as the unidirectional transfer of substances or signals between structural units of the system in its functional organization (Chauvet, 2002).

\section{Is Sentience in Artifacts Possible?}

Sentience is a minimalistic way of defining artificial consciousness without the requirement for biological laws. Complete emulation of the brain will be unable to produce sentient robots or any other kind of sentient system because to claim that, the brain computes is to show that it performs computations and many of its functions can clearly be represented algorithmically. However, "sentience" refers to the raw subjective perceptual experience which is noncomputable even when the performance of all the relevant functions is explained through algorithms. This is because sentience cannot be algorithmically represented through information theory and a "brain in a supercomputer" model based on information theory and computation is not going to yield the integration across scale that is needed to faithfully reproduce sentience for the reason that the topology included in such a model would be comprised. In other words, for subjective experiences to be actualized, there is an ingredient that such "brain in a supercomputer" could never possess - the topological nature of neural systems. The stabilizing nonsymmetric functional interactions define the topology of the system being modeled.

Consciousness is not reproducible in silicon or any other nonliving matter, since the ecology for consciousness must follow biological laws as opposed to physical laws. Consciousness requires energy and the complex energy field interactions would differ in nonliving matter. The interaction of biophotons within exciplex states of DNA is inclusively occurring in living matter and there is no guarantee that such can be effectively reproduced in man-made quantum computing machines. As such any theory that suggests consciousness is integration of information (Tononi, 2004) based on identity theory of problematic materialism undermines the tenets of field theory of integrative neuroscience. Consciousness as an integrator of information is flawed conceptually because consciousness is not a process and is non-computational so consciousness as a biological phenomenon cannot be integrated or be a physical entailment of an integration process as suggested by integration of information theory. The framework of the integrated information theory of consciousness (Balduzzi \& Tononi, 2008) is based on discrete dynamical systems which is totally incompatible with the integration process that is supposed to lead to a biophoton 
theory of consciousness. If the only reason why consciousness is not reproducible in silicon is due to the complexity of nonliving matter, then it should be possible in the near future to extract silicon from rocks and with precision engineering this component can participate in a machine that displays artificial intelligence by performing Boolean operations in a process that is called computation. In the fabrication of conscious subjective experiences in silicon, assuming the detailed complexity of brain operations are known a priori, the machine would also be required to implement biological laws in a process that is called biological computation. This is of course false since the functioning of our brains differs fundamentally from information theoretic consciousness as we have shown herein.

Over half a century ago, Eccles (1952) considered the brain to be a machine operating not according to biological laws, but the laws of physics and chemistry where a "ghost" operated the machine resulting in a dualist mind-brain liaison. Recently, the computational doctrine that the human brain is an information processing system and that thinking is a form of computing has been largely discredited (Hales, 2011; Manzotti, 2012). Conscious sentient awareness as a computation (Dehaene et al., 2014) is just as fallible. According to Reeke \& Edelman (1995) sentient artifacts may be possible, but they will certainly not be computers that process information. When should physical processes give rise to sentience? Pereira Jr (2013) claims that subjectivity arises with a kind of natural phenomenon that can be found in many natural substrates, such as ionic solutions, energy waves and nylon strings that exhibit "temporal" amplitude modulation. In brain activity, there is an important split of amplitude-modulated (AM) processes in time and space. Neurons display temporal amplitude modulation only in dendritic (graded) potentials, not in axon (action) potentials. Patterns embodied in spatially distributed AM dendritic fields can be integrated by the astroglial network, since the latter displays large-scale waveforms (Pereira Jr., 2013). As a consequence of the above reasoning, once engineers insert a wavelike substrate in a machine, suitably connected with the computational machinery and being affected by the content of the processed information, they would bestow subjectivity upon the machine. The machine could construct a machine-like subjective experience of the world.

\section{Conclusion}

The emergence of consciousness is not a macro-quantum effect, involving superconductivity, super-fluidity, electromagnetic fields, Einstein-Bose condensation, superfluorescence, luminescence or quantum vibrations of microtubules. We proposed a physical basis of consciousness based on complex interactions of subcellular energy fields. In particular, the interaction between the electric field and DNA results in conformational changes to DNA molecules which guide the biophoton energy field in a way similar to photonic crystal structures that are an ideal substrate for a quantum effect to instantiate coherent biophotons in the vast majority of neurons in the brain that form a biophoton field, as a consequence of it being radiation of light there is 
near instantaneous communication throughout the brain — referred to as quantum coherence. The biophoton field is realized as a conscious field when it is actualized by self-awareness in the aconscious as a result of re-absorption of biophotons by exciplex states of DNA chromosomes acquiring unlimited information from metabolic activity which in turn produce regulative electrical fields as a "feedback" mechanism of energy transfer across proteins as a result of protein-ligand binding during proteinprotein communication. We clearly articulate why the brain is specifically privileged to support a biophoton field theory of consciousness.

The genomic-based energy field theory of consciousness removes the elusiveness of finding the mechanisms underlying consciousness-related binding (Revonsuo \& Newman, 1999). Since the uniqueness of frequency patterns of energy fields is a reflection of the DNA codon sequences braiding patterns. However, the physiology underlying the function of consciousness is incompletely understood. In this regard, we have gone about explaining the nature of subjectivity of brain function in terms of metabolic activity and energy transfer. In particular, subjectivity instantiates as a resonant phenomenon arising from interactions between biophotons with exciplex states of DNA resulting in metabolic energy transfer in and between macromolecular proteins as subcellular signaling governed by Davydov solitonic transmission leading to a functional field that encompasses selected subjective experiences that are actualized through functional integration as qualia.

Finally a triple-aspect monistic model was derived in order to cognize the incognizability of the "steam" of consciousness in our quest to solve the hard problem of consciousness. In our endeavor, we have postulated that interactionism and not information theoretic views based on computation govern subjectivity in higherorder brain functioning. This can be viewed as an extension of the non-computational cognitive neuroscience paradigm pioneered by Globus (1992), while it certainly does satisfy the criteria for such a viewpoint, further research will be required to experimentally verify our claims of a genomic instantiation of consciousness in neurons through a biophoton field theory.

\section{Appendix: Definitions of Some Terms}

Aconscious - refers to the unconscious that is not repressed nor directly retrievable to consciousness.

Biophotons - is a photon of non-thermal origin emitted from a biological system or ultra-weak biological photon emissions; photons are massless elementary particles of null charge.

Biophoton emission - is the release of coherent photons from cells as a process of radiative relaxation of electro-solitons. Non-coherent release can be from exciplex states of DNA or as byproduct of metabolism.

Causality - (also referred to as causation) is the relation between the cause and the effect where the effect is understood as a consequence of the cause. 
Cognitive function - is a brain function recalled to consciousness via psychophysiological storage embedded in the functional organization and causally reducible to cognitive semantics.

Cognitive semantics - is the study of meaning. It focuses on the relation between signifiers, like words, phrases, signs, and symbols, and what they stand for, their denotation.

Complex adaptive system - consists of interactions that govern the complex dynamics through biological laws whose collective behavior exhibits emergent properties, i.e., the interactions lead to new properties of the system as a whole.

Cognized function - is a brain function causally reducible to cognitive processes.

Conformational change - a transition between macromolecular conformations induced by many factors.

Consciousness - is a physical state of self-awareness realized by quantum coherence in the brain and actualized by DNA re-absorption of biophotons and the resultant effect of energy transfer across proteins by protein-ligand binding in neurons.

Conscious function - is a cognized function arising in the aconscious within the functional organization that has been recalled to consciousness.

Darwinian process during ontogenesis - self-organizing process based on selection of random variation.

Degeneracy - is an evolution theory where relational property between two or more structures where functional redundancy facilitates evolution.

Developmental selectionism - Darwinian processes carried out in the brain during ontogenesis.

Davydov soliton - a molecular soliton that can be used as a dissipative soliton in the sense that on a short time scale dissipation of spatial transfer of vibrational energy can be neglected, and on a long scale the amplitude of the soliton will decay and finally vanish.

Dynamic adaptability - adaptability of dynamical pressures selected from either environmental influence (i.e., stimuli), past experience or behavior (i.e., a form of learning) leading toward the formation of self-referential qualities.

Dynamic continuity - continuous spatiotemporal patterns of information flow carried by the electric energy field inside neurons and across synapses (e.g., as a result of a change in the electrochemical signature at an appropriate hierarchical level).

Dynamic connectivity - a dynamic association of neurons under chemical and cytoplasmic pressures leading to the formation of neuronal groups or assemblies through the alternation of dynamic continuity by changes to synaptic efficiency and hence synaptic connectivity.

Electro-solitons - solitons that carry an electric charge (i.e., an electric energy wave field).

Emotions - are the physiological and behavioral responses to subjective conscious experiences (or qualia).

Entropy - is an organizational change in a physical system. 
Entanglement - the phenomenon of two or more segregated entities sharing a brain state.

Exciplex state - short lived complex formed between different molecules when one is excited by a biophoton.

Explicit specification — morphogenesis leading to function determined by a preexisting plan where each neuron has a specific "address" guided by extracellular forces and the DNA in cell nucleus.

Feedback - interactions occur in both directions within the brain and between the brain and its environment.

Functionalism - suggests that structural changes may not entail functional changes and requires a holistic view of functions as being separate (i.e., independent of structure) and/or not supervening (i.e., come from the structure in all aspects).

Functional interaction — is a dynamic interaction which leads to a particular fuctional role; it is also defined mathematically as three elements: (i) the source, (ii) the sink and (iii) the transformation within the sink that possesses nonsymmetry and non-locality properties.

Functional field - is a specific grouping of neural systems in which chemical reactions associated with metabolic activity arise from the dynamical interactions in the brain.

Functional integration — is the resultant effect of specification of qualia during the integration of functional interactions.

Functional organization - mapping as a result of functional integration at various hierarchical levels (each hierarchical level of organization is assumed to be locally connected to the next higher level).

Incognizable - a form of dynamical interaction (not a brain function) that is causally irreducible to cognitive processes.

Intentionality — is the ability to have thoughts "about" something.

Hard problem - is how the subjective-experience can be described objectively.

Macroergic effects - collective interactions leading to electronic excitations in macromolecules.

Metastability - a transitory epoch in which an initial configuration coalesces into a final configuration.

Nonreductive functionalism — subjective experiences can only be defined nonreductively, in terms of the general pattern of their interactions with one another i.e., subjective experiences arise from integration of functional interactions and not from individual functions.

Ontology - mode of existence.

Qualia - is a conscious function that is a quantum phenomenon of raw sensation of subjective conscious experiences (sing. Quale).

Re-entry - refers to the inter connectivity and exchange of signals between neuronal groups. 
Relational organization - includes all relations between material parts, relations between the effects of interactions of material parts, and relations with time and environment.

Reductionism - one-way process of reduction whereby global phenomena can be understood to be a collection of various hierarchically linked local phenomena that affect the overall global phenomena.

Relational organization - mapping as a result of integration of dynamical interactions at various hierarchical levels (each hierarchical level of organization is assumed to be locally connected to the next higher level).

Resonance - is the optimum change to a functional organization of a neural system in which transfer of excitation occurs naturally.

Segregation - we can interpret it as in embryology, where the differentiation of a new structure still retains the unity with the whole.

Sentience - is the ability of any entity to have subjective perceptual experiences, without necessity of biological laws.

Selectionism — is synonymous with evolution by natural selection (or Darwin's theory of natural selection).

Self-awareness — is an immediate subjective sense of being; that is, a moment-bymoment sense of one's existence, a sense one is alive, that one is awake; this can be equated with what is consciousness.

Self-organization - process where random or non-algorithmic changes manifest themselves into a coherent organized activity (i.e., synergetics).

Self-referential organization — the ontological embodiment of epistemological aspects of a complex adaptive system.

Subjective conscious experience - is a quantum phenomenon of biophoton field interactions appearing when the aconscious subjective experience is recalled to consciousness and therefore is actualized.

Subjective experience - is a quantum phenomenon of biophoton field interactions and the realizer of functional field brought about during the functional integration arising from resonant dynamical interactions in the brain.

Supervenience — is used to describe cases where properties (i.e., often structure dependent) of a system are consequent on the existence or establishment of another (i.e., can be traced back "a posteriori to the structure).

Synaptic connectivity - a static and physical association of neurons connected through chemical synapses generated.

Synaptic plasticity - changes in transmitter release or biophysical properties of the synapse as a result of correlated activity between pre- and post-synaptic cells (also a kind of Hebb synapse).

Uncognized function - is a brain function arising in the aconscious within the self-referential organization that is not represented and has not been recalled to consciousness. 


\section{Acknowledgments}

The authors appreciate detailed discussions with Dorian Aur, Alfredo Pereira Jr., George N. Reeke, Jr. and Ram L.P. Vimal.

\section{REFERENCES}

Aur, D. \& Jog, M. (2010) Neuroelectrodynamics: Understanding the Brain Language. Netherlands: IOS Press.

Aur, D., Jog, M. \& Poznanski, R.R. (2011) Computing by physical interaction in neurons. J. Integr. Neurosci., 10, 413-422.

Bauer, R. \& Dicke, P. (1997) Fast cortical selection: A principle of neuronal self-organization for perception? Biol. Cybern., 77, 207-215.

Balduzzi, D. \& Tononi, G. (2008) Integrated information in discrete dynamical systems: Motivation and theoretical framework. PLoS Comput. Biol., 4, e1000091.

Balduzzi, D. \& Tononi, G. (2009). Qualia: The geometry of integrated information. PLoS Comput. Biol., 5, e1000462.

Block, N. (1994) Qualia. In: S. Guttenplan, ed. A Companion to Philosophy of Mind. Oxford: Blackwell Publishers.

Breakspear, M. (2004) Dynamic connectivity in neural systems. Neuroinformatics, 2, 205-224.

Brizhik, L. (2008) Nonlinear mechanism for weak photon emission from biosystems. Indian J. Exp. Biol., 46, 353-357.

Brizhik, L., Cruzeiro-Hansson, L. \& Eremko, A. (1998) Influence of electromagnetic radiation on molecular solitons. J. Biol. Phys., 24, 19-39.

Bruzzo, A.A. \& Vimal, R.L.P. (2007) Self: An adaptive pressure arising from self-organization, chaotic dynamics, and neural Darwinism. J. Integr. Neurosci., 6, 541-566.

Bullock, T.H. (1993) Circuitry and beyond: How far does connectivity get us? In: How Do Brains Work?: Papers of a Comparative Neurophysiologist. Boston: Birkhauser.

Bullock, T.H. (1997) Signals and signs in the nervous system: The dynamic anatomy of electrical activity is probably information-rich. Proc. Natl. Acad. Sci. USA, 94, 1-6.

Buzsaki, G., Anastassiou, C.A. \& Koch, C. (2012) The origin of extracellular fields and currents-EEG, ECoG, LFP and spikes. Nat. Rev. Neurosci., 13, 407-420.

Cacha, L.A. \& Poznanski, R.R. (2011) Associable representations as field of influence for dynamic cognitive processes. J. Integr. Neurosci., 10, 423-437.

Calvin, W.H. (1998) Competing for consciousness: A Darwinian mechanism at an appropriate level of explanation. J. Conscious. Stud., 5, 389-404.

Caroni, P. (1998) Driving the growth cone. Science, 281, 1465-1466.

Carruthers, P. \& Veillet, B. (2007) The phenomenal concept strategy. J. Conscious. Stud., 14, 212-236.

Chalmers, D.J. (1993) Toward a theory of consciousness. Unpublished Ph.D, thesis. Indiana University, Bloomington.

Chalmers, D.J. (1995) Facing up to the problem of consciousness. J. Conscious. Stud., 2, 200219.

Chalmers, D.J. (1996) The Conscious Mind: In Search of a Fundamental Theory. NY: Oxford University Press. 
Chalmers, D.J. (2006) Strong and weak emergence. In: P. Clayton and P. Davis, eds. The Re-Emergence of Emergence. New York: Oxford University Press.

Chauvet, G.A. (1993a) Non-locality in biological systems results from hierarchy: A pplication to the nervous system. J. Math. Biol., 31, 475-486.

Chauvet, G.A. (1993b) Hierarchical functional organization of formal biological systems: A dynamical approach. I. An increase of complexity by self-association increases the domain of stability of a biological system. Phil. Trans. Roy. Soc. Lond. B, 339, 425-444.

Chauvet, G.A. (1993c) Hierarchical functional organization of formal biological systems: A dynamical approach. II. The concept of non-symmetry leads to a criterion of evolution deduced from an optimum principle of the (O-FBS) sub-system. Phil. Trans. R. Soc. Lond. $B, 339,445-461$.

Chauvet, G.A. (1993d) Hierarchical functional organization of formal biological systems: A dynamical approach. III. The concept of non-locality leads to a field theory describing the dynamics at each level of organization of the (D-FBS) sub-system. Phil. Trans. R. Soc. Lond. B, 339, 463-481.

Chauvet, G.A. (1996) Theoretical Systems in Biology: Hierarchical \& Functional Integration. Oxford: Pergamon Press.

Chauvet, G.A. (2002) On the mathematical integration of the nervous tissue based on the S-propagator formalism. I. theory. J. Integr. Neurosci., 1, 31-68.

Chauvet, G.A. (2004) The Mathematical Nature of the Living World. The Power of Integration. Singapore: World Scientific Publishers.

Cifra, M., Fields, J. Z \& Farhadi, A. (2011) Electromagnetic cellular interactions. Prog. Biophysics. Mol. Biol., 105, 223-246.

Crick, F. (1970) Central dogma of molecular biology. Nature, 227, 561-563.

Crick, F. (1990) Neural Edelmanism: Reply. Trends Neurosci., 13, 13-14.

Davia, C.J. (2006) Life, catalysis and excitable media: A dynamic systems approach to metabolism and cognition. In: J. Tuszynski, ed. The Emerging Physics of Consciousness. Heidelberg, Germany: Springer-Verlag.

Davydov, A.S. (1973) The theory of contraction of proteins under their excitation. J. Theor. Biol., 38, 559-569.

Davydov, A.S. (1991) Solitons in Molecular Systems. 2nd edn. Dordrecht: Kluwer Academic Publishers.

Dehaene, S., Charles, L., King, J.-R. \& Marti, S. (2014) Toward a computational theory of conscious processing. Curr. Opin. Neurobiol., 25, 76-84.

Dennett, D. C (1991) Consciousness Explained. Boston, MA: Little Brown.

Dennis, M. Y, Nuttle, X., Sudmant, P.H., Antonacci, F., Graves, T.A., Nefedov, M., Rosenfeld, J.A., Sajjadian, S., Malig, M., Kotkiewicz, H., Curry, C.J., Shafer, S., Shaffer, L.G., deJong, P.J., Wilson, R. \& Eichier, E.E. (2012) Evolution of human-specific neural SRGAP2 genes by incomplete segemental duplication. Cell, 149, 912-922.

Eccles, J.C. (1952) The Neurophysiological Basis of Mind. Oxford: Clarendon Press.

Edelman, G.M. (1978) Group selection and phasic re-entrant signaling: A theory of higher brain function. In: G. M. Edelman and V. B. Mountcastle, eds. The Mindful Brain: Cortical Organization and the Group-Selective Theory of Higher Brain Function. Cambr., MA: The MIT Press. 
Edelman, G.M. (1981) Group selection as the basis for higher brain function. In: F. O. Schmitt, F. G. Worden, G. Adelman and S. G. Dennis, eds. The Organization of the Cerebral Cortex. Cambr., MA: The MIT Press.

Edelman, G.M. (1987) Neural Darwinism: The Theory of Neuronal Group Selection. New York: Basic Books.

Edelman, G.M. (1993) Neural Darwinism: Selection \& re-entrant signaling in higher brain function. Neuron, 10, 115-125.

Edelman, G.M. (1995) Memory and the individual soul: Against silly reductionism. In: J. Cornwell, ed. Nature's Imagination: The Frontiers of Scientific Vision. Press, Oxford: Oxford University.

Edelman, G.M. \& Gally, J.A. (2001) Degeneracy and complexity in biological systems. Proc. Natl. Aced. Sci. USA, 98, 13763-13768.

Edelman, G.M. \& Tononi, G. (1995) A Universe of Consciousness: How Matter Becomes Imagination. New York: Basic Books.

Eliasmith, C., Stewart, T.C., Choo, X., Bekolay, T., De Wolf, T. Tang, Y. \& Rasmussen, D. (2012) A large-scale model of the functioning brain. Science, 338, 1202-1205.

Edwards, J.C.W. (2005) Is consciousness only a property of individual cells. J. Conscious. Stud., 12, 60-76.

Feinberg, T.E. (2012) Neuroontology, neurobiological naturalism, and consciousness: A challenge to scientific reduction and a solution. Phys. Life. Rev., 9, 13-34.

Feinberg, T.E. \& Mallatt, J. (2013) The evolutionary and genetic origins of consciousness in the Cambrian period over 500 million years ago. Front. Psychol., 4, 667.

Fingelkurts, A.A. \& Fingelkurts, A.A. (2004) Making complexity simpler: Multivariability and mestability in the brain. Int. J. Neurosci., 114, 843-862.

Fingelkurts, A.A., Fingelkurts, A.A. \& Neves, C.F.H. (2009) Phenomenological architecture of a mind and operational architectonic of the brain: The unified metastable continuum. New Math. Nat. Comput., 5, 221-244.

Frank, E. \& Wenner, P. (1993) Environmental specification of neuronal connectivity. Neuron, 10, 779-785.

Freeman, W.J. (2005) NDN, volume transmission, and self-organization in brain dynamics. J. Integr. Neurosci., 4, 407-421.

Frank-Kamenetskii, M.D. \& Lazurkin, Y.S. (1974) Conformational changes in DNA molecules. Annu. Rev. Biophys. BioEng., 3, 127-150.

Freeman, W.J. (2007) Indirect biological measures of consciousness from field studies of brains as dynamical systems. Neural Netw., 20, 1021-1031.

Freeman, W.J. \& M.D. Holmes (2005) Metastability, instability, and state transitions in neocortex. Neural Netw., 18, 497-504.

Freud, S. (1915) The Unconscious. The Standard Edition of the Complete Psychological Works of Sigmund Freud. Vol. 14. London: Hogarth Press, pp. 166-204.

Fröhlich, H. (1968) Long-range coherence and energy storage in biological systems. J. Quantum Chem., 2, 641-649.

Fuxe, K. \& Agnati, L.F. (eds.) (1991) Volume Transmission in the Brain: Novel Mechanisms for Neural Transmission. New York: Raven Press.

Gierer, A. (1988) Spatial organization and genetic information in brain development. Biol. Cybern., 59, 13-21. 
Giudice, E.D., Preparata, G. \& Vitiello, G. (1988) Water as a free electric dipole laser. Phys. Rev. Lett., 61, 1085-1088.

Globus, G.G. (1992) Towards a noncomputational cognitive neuroscience. J. Cognit. Neurosci., 4, 299-310.

Golant, M.B. (1989) Resonance effect of coherent electromagnetic radiation of millimetre range of waves on the living organisms. Biofizika, 34, 1004-1014.

Goodman, G. (1983) Light penetration of the cranium of the male domestic fowl in artificial and natural conditions. Unpublished Ph.D. dissertation. Hebrew University of Jerusalem, Israel.

Green, H.S. \& Triffet, T. (1997) Source of Consciousness: The Biophysical and Computational Basis of Thought. World Scientific Publishers, Singapore.

Grossberg, S. (1999) The link between brain learning, attention, and consciousness. Conscious. Cognit., 8, 1-44.

Grossberg, S. (2007) Consciousness CLEARS the mind. Neural Netw., 20, 1040-1053.

Haken, H. (1996) Principles of Brain Functioning: A Synergetic Approach to Brain Activity, Behavior and Cognition. Berlin: Springer-Verlag.

Hameroff, S.R. (1994a) Quantum coherence in microtubules: A neural basis for emergent consciousness? J. Conscious. Stud., 1, 98-118.

Hameroff, S.R. (1994b) Toward a Scientific Basis for Consciousness. Cambridge, MA: MIT Press.

Hameroff, S.R. \& Penrose, R. (2014) Consciousness in the universe: A review of the 'Orch OR' theory. Phys. Life Rev., 11, 39-78.

Harris-Warrick, R.M. \& Marder, E. (1991) Modulation of neural networks for behavior. Annu. Rev. Neurosci., 14, 39-57.

Haugeland, J. (1980) Programs, causal powers, and intentionality (comment on Searle). Behav. Brain Sci., 3, 432-433.

Hales, C. (2011) On the status of computationalism as a law of nature. Int. J. Machine Conscious., 3, 55-90.

Isojima, Y., Isoshima, T., Nagai, K., kikuchi, K. \& Nakagawa, H. (1995) Ultraweak biochemiluminescence detected from rat hippocampal slices. Neuroreport, 6, 658-660.

Izhikevich, E.M. \& Edelman, G.M. (2008) Large-scale model of mammalian thalamocortical systems. Proc. Nat. Acad. Sci. USA, 105, 3593-3598.

Jirsa, V.K. \& McIntosh, A.R. (eds.) (2007) Handbook of Brain Connectivity. Berlin: Springer.

John, E.R. (2001) A field theory of consciousness. Conscious. Cognit., 10, 184-213.

Kataoka, Y., Cui, Y., Yamagata, A., Niigaki, M., Hirohata, T., Oishi, N. \& Watanabe, Y. (2001) Activity-dependent neural tissue oxidation emits intrinsic ultraweak photons. Biochem. Biophys. Res. Commun., 285, 1007-1011.

Kelso, J.A.S. (1995) Dynamic Patterns: The Self-organization of Brain and Behaviour. Cambridge, MA: MIT Press.

Kim, J. (1998) Mind in a Physical World: An Essay on the Mind-Body Problem and Mental Causation. MIT Press, Cambridge, MA.

Kim, J. (2009). Mental causation. In: B. McLaughlin, A. Beckermann and S. Walter eds. The Oxford Handbook of Philosophy of Mind. Oxford Handbooks Online.

Kobayashi, M., Takeda, M., Sato, T., Yamazaki, Y., Kaneko, K., Ito, K., Kato, H. \& Inaba, H. (1999) In vivo imaging of spontaneous ultraweak photon emissions from a rat's 
brain correlated with cerebral energy metabolism and oxidative stress. Neurosci. Res., 34, 103-113.

LaBerge, D. \& Kasevich, R. (2007) The apical dendrite theory of consciousness. Neural Netw., 20, 1004-1020.

Levin, J. (2006). What is a phenomenal concept? In: T. Alter and S. Walter (eds.), Phenomenal Concepts and Phenomenal Knowledge. New Essays on Consciousness and Physicalism. Oxford: Oxford University Press, pp. 87-110.

Levin, J. (2008). Taking type-B materialism seriously. Mind Lang., 23, 402-425.

Levine, J. (1983). Materialism and qualia: The explanatory gap. Pac. Philos. Quart., 64, 354-361.

Li, K.H. (1992) Coherent radiation from DNA molecules. In: F. A. Popp, K. H. Li and Q. Gu, eds. Recent Advances in Biophoton Research and Its Applications. Singapore: World Scientific.

Libet, B.J. (1996) Conscious mind as a field. J. Theor. Biol., 178, 223-243.

Lindahl, B.I.B. \& Arhem, P. (1994) Mind as a force field: Comments on a new interactionistic hypothesis. J. Theoret. Biol., 181, 95-96.

Lindsay, K.A., Rosenberg, J.R. \& Tucker, G. (2004) From Maxwell's equations to the cable equation and beyond. Prog. Biophys. Molec. Biol., 85, 71-116.

Llinas, R., Ribary, V., Contreras, D. \& Pedroarena, C. (1998) The neuronal basis for consciousness. Phil. Trans. R. Soc. B, 353, 1841-1849.

MacGregor, R.J. (2006) On the Contexts of Things Human: An integrative View of Brain, Consciousness, and Freedom of Will. Singapore: World Scientific.

Manzotti, R. (2012) The computational stance is unfit for consciousness. Intern. J. Machine Conscious. 4, 401-420.

McCarty, M. (1985) The Transforming Principle: Discovering that Genes are Made of DNA., New York: W.W. Norton.

McFadden, J. (2002a) Synchronous firing and its influence on the brain's electromagnetic field: Evidence for an electromagnetic theory of consciousness. J. Conscious. Stud., 9, 23-50.

McFadden, J. (2002b) The conscious electromagnetic information(Cemi) field theory: The hard problem made easy? J. Conscious. Stud., 9, 45-60.

McFadden, J. (2013) The CEMI field theory closing the loop. J. Conscious. Stud., 20, $153-168$.

McGinn, C. (1995) Consciousness and space. J. Conscious. Stud., 2, 220-230.

Merker, B. (2007) Consciousness without a cerebral cortex: A challenge for neuroscience and medicine. Behav. Brain Sci., 30, 63-81.

Orpwood, R.D. (2007) Neurobiological mechanisms underlying qualia. J. Integr. Neurosci., 6, 523-540.

Orsucci, F. (2009) Mind Force. On Human Attractions. Singapore: World Scientific Publishers.

Pereira Jr. A. (2013) Triple-aspect monism: A conceptual framework for the science of human consciousness. In: A. Pereira Jr. and D. Lehmannm, eds. The Unity of Mind, Brain and World: Current Perspectives on a Science of Consciousness. Cambridge University Press: Cambridge, UK.

Penrose, R. (1995) Consciousness involves noncomputable ingredients. In: J. Brockman, ed. The Third Culture: Beyond the Scientific Revolution. New York: Simon and Schuster. 
Peressini, A. (2013). Consciousness as integrated information: A provisional philosophical critique. J. Conscious. Stud., 20, 180-206.

Popp, F.A. \& Chang, J.J. (1998) The physical background and the informational character of biophoton emission. In: J.J. Chang, J. Fish and F.A. Popp, eds. Biophotons. Dordrecht: Kluwer.

Popp, F.A., Chang, J.J., Herzog, A., Yan, Z. \& Yan, Y. (2002) Evidence of non-classical (Squeezed) light in biological systems. Phys. Lett. A, 293, 98-102.

Popp, F.A., Gu, Q. \& Li, K.-H. (1994) Biophoton emission: Experimental background and theoretical approaches. Mod. Phys. Let. B, 8, 1269-1296.

Popp, F.A., Nagl, W., Li, K.H., Scholz, W., Weingartner, O. \& Wolf, R. (1984) Biophoton emission: New evidence for coherence and DNA as source. Cell Biophys., 6, 33-52.

Popper, K., Lindahl, B.I.B. \& Arhem, P. (1993) A discussion on the mind-body problem. Theor. Med., 14, 167-180.

Poznanski, R.R. \& Cacha, L.A. (2011) Intracellular capacitive effects of polarized proteins in dendrites. J. Integr. Neurosci., 11, 417-438.

Rahnama, M., Tuszynski, J.A., Bokkon, I., Cifra, M., Sardar, P. \& Salari, V. (2011) Emission of mitochondrial biophotons and their effect on electrical activity of membrane via microtubules. J. Integr. Neurosci., 10, 65-88.

Rattemeyer, M., Popp, F.A. \& Nagl, W. (1981) Evidence of photon emission from DNA in living systems. Naturwissen, 68, 572-580.

Rashevsky, N. (1961) Mathematical Principles in Biology and Their Applications. Springfield, IL: Charles C. Thomas.

Reeke, Jr., G.N. (1994) Selection versus instruction: Use of computer models to compare brain theories. Int. Rev. Neurobiol., 37, 211-241.

Reeke, Jr. G.N. \& Edelman, G.M. (1995) A Darwinist view of the prospects for conscious artifacts. In: G. Trautteur, ed. Consciousness: Distinction and Reflection. Naples: Bibliopolis.

Reeke, Jr., G.N. \& Sporns, O. (1990) Selectionist models of perceptual and motor systems and implications for functionalist theories of brain function. Physica D, 42, 347-364.

Reeke, Jr., G.N., Sporns, O. \& Edelman, G.M. (1990) Synthetic neural modeling: The "Darwin" series of recognition automata. Proc. IEEE, 78, 1498-1530.

Revonsuo, A. \& Newman, J. (1999) Binding and consciousness. Conscious. Cogn., 8, $123-127$.

Ricciardi, L.M. \& Umezawa, H. (1967) Brain and physics of many body problems. Kybernetik, 4, 44-48

Rolls, E.T. (1997) Consciousness in neural networks. Neural Netw., 10, 1227-1240.

Rosen, R. (1958) A relational theory of biological systems. Bull. Math. Biophys., 20, 245-260.

Rosen, R. (1991) Life Itself: A Comprehensive Inquiry into the Nature, Origin, and Fabrication of Life. New York: Columbia University Press.

Rosen, R. (1998) Essays on Life Itself. New York: Columbia University Press.

Sataric, M.V., Tuszynski, J.A. \& Zakula, R.B. (1993) Kinklike excitations as an energytransfer mechanism in microtubules. Phys. Rev E., 48, 589-597.

Sataric, M.V., Zakula, R.B. \& Tuszynski, J.A. (1992) A model of the energy transfer mechanism in microtubules involving a single soliton. Neurobiol., 1, 445-456.

Schrödinger, E. (1944) What is Life?: The Physical Aspects of the Living Cells. Cambridge: Cambridge University Press. 
Scott, A.C. (1985) Biological solitons. In: S. Takeno, ed. Dynamical Problems in Soliton Systems. Berlin: Springer-Verlag.

Searle, J.R. (2000) Consciousness. Annu. Rev. Neurosci., 23, 557-578.

Searle, J.R. (2007) Biological naturalism. In: M. Velmans and S. Schneider, eds. The Blackwell Companion to Consciousness., Oxford: Blackwell.

Seth, A.K., Izhikevich, E., Reeke, G.N. \& Edelman, G.M. (2006) Theories and measures of consciousness: An extended framework. Proc. Natl. Acad. Sci. USA, 103, 10799-10804.

Sevush, S. (2006) Single-neuron theory of consciousness. J. Theor. Biol., 238, 704-725.

Shewmon, D. A., Holmes, G. L. \& Byrne, P.A. (1999) Consciousness in congenitally decorticate children: Developmental vegetative state as self-fulfilling prophecy. Dev. Med. Child Neurol., 41, 364-374.

Smith, C.U.M. (2006) The 'hard problem' and the quantum physicists. Part 1: The first generation. Brain Cogni., 61, 181-188.

Smith, C.U.M. (2009) The 'hard problem' and the quantum physicists. Part 2: Modern times. Brain Cogni., 71, 54-63.

Solms, M. \& Turnbull, O. (2002) Brain and the Inner World: An Introduction to the Neuroscience of Subjective Experience. New York: Other Press.

Snyder, A.W., Mulcahy, E., Taylor, J.L., Mitchell, D.J., Sachdev, P. \& Gandevia, S.C. (2003) Savant-like skills exposed in normal people by suppressing the left fronto-temporal lobe. J. Integr. Neurosci., 2, 149-158.

Sporns, O. (1997) Deconstructing neural constructivism. Behav Brain Sci., 20, 576-577.

Sporns, O. \& Tononi, G. (eds.) (1994) Selectionism and the Brain. New York: Academic Press.

Stenger, V.J. (1993) The myth of quantum consciousness. Humanist, 53, 13-15.

Stewart, T.C. \& Eliasmith, C. (2014) Large-scale synthesis of functional spiking neural circuits. Proc. IEEE, 102, 881-898.

Sun, Y. Wang, Ch. \& Dai, J. (2010) Biophotons as neural communication signals demonstrated by in situ biophoton autography. Photochem. Photobiol. Sci., 9, 315-322.

Taylor, J.G. (2009) Modeling consciousness. New Math. Nat. Comp., 5, 31-46.

Tegmark, M. (2000) Importance of quantum decoherence in brain processes. Phys. Rev. E., 61, 4194-4206.

Thar, R. \& Kuhl, M. (2004) Propagation of electromagnetic radiation in mitochondria? J. Theor. Biol., 230, 261-270.

Tononi, G. (2004) An information integration theory of consciousness. BMC Neurosci., $\mathbf{5}, 42$.

Tononi, G. (2008) Consciousness as integrated information: A provisional manifesto. Biol Bull., 215, 216-242.

Tononi, G. (2010) Information integration: Its relevance to brain function and consciousness. Arch. Ital. Biol., 148, 299-322.

Tononi, G. (2012) Integrated information theory of consciousness: An updated account. Arch. Ital. Biol., 150, 293-329.

Tononi, G. \& Sporns, O. (2003) Measuring information integration. BMC Neurosci., 4, 31.

Tononi, G. \& Edelman, G.M. (1998) Consciousness and complexity. Science, 282, 1846-1851.

Turton, D.A., Senn, H.M., Harwood, T., Lapthorn, A.J., Ellis, E.M. \& Wynne, K. (2014) Terahertz underdamped vibrational motion governs protein-ligand binding in solution. Nat. Commun., 5, 3999. 
Tuszynski, J.A. (ed.) (2006) The Emerging Physics of Consciousness. Berlin: Springer-Verlag. Tuszynski, J.A., Paul, R., Chatterjee, R. \& Sreenivasa, S.R. (1984) Relationship between Fröhlich and Davydov models of biological order. Phys. Rev. A, 30, 2666-2675.

Van Wilk, R., Godaerts, G. \& Van Wijk, E.P.A. (2007) Human ultra weak light emission in consciousness research, In: J.T. Locks, ed. New Research on Consciousness. New York: Nova Science Publishers.

Vimal, R.L.P. (2008) Proto-experiences and subjective experiences: Classical and quantum concepts. J. Integr. Neurosci., 7, 49-73.

Vimal, R.L.P. (2010) Matching and selection of a specific subjective experience: Conjugate matching and subjective experience. J. Integr. Neurosci., 9, 193-251.

Watson, J.D. \& Crick, F.H. (1953) Molecular structure of nucleic acids. A structure for deoxyribose nucleic acid. Nature, 171, 737-738.

Willshaw, D.J \& Malsburg, C. von der (1976) How patterned neural connections can be setup by self-organization. Proc. R. Soc. Lond B, 194, 431-445. 